\title{
Genome-wide comparative analysis of NBS-encoding genes between Brassica species and Arabidopsis thaliana
}

Jingyin $\mathrm{Yu}^{1 \dagger}$, Sadia Tehrim ${ }^{1 \dagger}$, Fengqi Zhang ${ }^{1}$, Chaobo Tong ${ }^{1}$, Junyan Huang ${ }^{1}$, Xiaohui Cheng ${ }^{1}$, Caihua Dong ${ }^{1}$, Yanqiu Zhou, ${ }^{1,2}$ Rui Qin², Wei Hua ${ }^{1}$ and Shengyi Liu' ${ }^{1 *}$

\begin{abstract}
Background: Plant disease resistance (R) genes with the nucleotide binding site (NBS) play an important role in offering resistance to pathogens. The availability of complete genome sequences of Brassica oleracea and Brassica rapa provides an important opportunity for researchers to identify and characterize NBS-encoding $R$ genes in Brassica species and to compare with analogues in Arabidopsis thaliana based on a comparative genomics approach. However, little is known about the evolutionary fate of NBS-encoding genes in the Brassica lineage after split from A. thaliana.

Results: Here we present genome-wide analysis of NBS-encoding genes in B. oleracea, B. rapa and A. thaliana. Through the employment of HMM search and manual curation, we identified 157, 206 and 167 NBS-encoding genes in B. oleracea, B. rapa and A. thaliana genomes, respectively. Phylogenetic analysis among 3 species classified NBS-encoding genes into 6 subgroups. Tandem duplication and whole genome triplication (WGT) analyses revealed that after WGT of the Brassica ancestor, NBS-encoding homologous gene pairs on triplicated regions in Brassica ancestor were deleted or lost quickly, but NBS-encoding genes in Brassica species experienced species-specific gene amplification by tandem duplication after divergence of B. rapa and B. oleracea. Expression profiling of NBS-encoding orthologous gene pairs indicated the differential expression pattern of retained orthologous gene copies in $B$. oleracea and B. rapa. Furthermore, evolutionary analysis of CNL type NBS-encoding orthologous gene pairs among 3 species suggested that orthologous genes in B. rapa species have undergone stronger negative selection than those in $B$.oleracea species. But for TNL type, there are no significant differences in the orthologous gene pairs between the two species.

Conclusion: This study is first identification and characterization of NBS-encoding genes in B. rapa and B. oleracea based on whole genome sequences. Through tandem duplication and whole genome triplication analysis in $B$. oleracea, B. rapa and A. thaliana genomes, our study provides insight into the evolutionary history of NBS-encoding genes after divergence of $A$. thaliana and the Brassica lineage. These results together with expression pattern analysis of NBS-encoding orthologous genes provide useful resource for functional characterization of these genes and genetic improvement of relevant crops.
\end{abstract}

Keywords: Brassica species, Disease resistance gene, Nucleotide binding site, Tandem duplication, Whole genome duplication

\footnotetext{
*Correspondence: liusy@oilcrops.cn

${ }^{\dagger}$ Equal contributors

'Key Laboratory of Biology and Genetic Improvement of Oil crops, the Ministry of Agriculture, Oil Crops Research Institute of the Chinese Academy of Agricultural Sciences, Wuhan 430062, China

Full list of author information is available at the end of the article
} 


\section{Background}

Plants are surrounded by a large number of invaders including bacteria, fungi, nematodes and viruses, and some of them have successfully invaded crop plants and cause diseases which result in deterioration of crop quality and yield. In order to cope with disease attacks, the plants have developed multiple layers of defense mechanisms. Plant disease resistance $(R)$ genes which specifically interact/recognize with corresponding pathogen avirulence (avr) genes are considered as plant genetic factors of a major layer. The interactions of this gene-for-gene (or genes-for-genes) manner activate the signal transduction cascades that turn on complex defense responses against pathogen attack and this is called incompatible interaction [1]. The interaction between a host species and a pathogenic species is dynamic where a host variety often lost the $\mathrm{R}$ gene-dependent resistance due to its pathogen race evolution for a virulent gene and thus a new $R$ gene was selected against this new race [2]. $R$ genes provide innate immunity whereas outcomes of defense responses lacking $R$ genes are partial resistance [3]. Therefore, identification of $\mathrm{R}$ genes is crucial for resistant variety development and relevant mechanism investigation.

To date, more than one hundred $\mathrm{R}$ genes, which was reported in PRGdb (http://prgdb.crg.eu/wiki), were functionally identified and comprise a super family in plants [4]. Sequence composition analysis of $R$ genes indicate that they share high similarity and contain seven different conserved domains like NBS (nucleotide-binding site), LRR (leucine rich repeat), TIR (Toll/Interleukin-1 receptor), CC (coiledcoil), LZ (leucine zipper), TM (transmembrane) and STK (serine-threonine kinase). Based on domain organization, $\mathrm{R}$ gene products can be categorized into five major types: TNL (TIR-NBS-LRR), CNL (CC-NBS-LRR), RLK (Receptor like kinases), RLP (Receptor like proteins) and Pto (a Ser/Thr kinase protein) [1,5,6]. Most of the $\mathrm{R}$ genes in plant kingdom are members of NBS-LRR (nucleotide-binding site-leucine rich repeat) proteins. 'NBS' and 'LRR' domains play different roles in plant-microbe interaction, where the former have the ability to bind and hydrolyze ATP or GTP and the latter is involved in protein-protein interactions [7]. NBS-LRR proteins in plants share sequence similarity with the mammalian NOD-LRR containing proteins which play a role in inflammatory and immune responses. On the basis of presence or absence of $\mathrm{N}$-terminal domains (TOLL/ interleukin-1 receptor (TIR) and the coiled-coil (CC) motif), NBS-LRR class can be further divided into two major types, TNL (TIR-NBS-LRR) and CNL (CC-NBS-LRR). TNL type share homology with the Drosophila toll and human interleukin-1 receptor (TIR). The two types show divergence in their sequence and signaling pathways. Several partial NBS-LRR variants like TIR, TIR-NBS (TN), CC, CC-NBS(CN) and NBS (N) have also been identified in plant species $[6,8,9]$.
Recent whole genome sequence data enabled the genome wide identification, mapping and characterization of candidate NBS-containing $\mathrm{R}$ genes in economically important plants. For example, the approximate arrays of 159 NBS-encoding R genes in A. thaliana [10], 581 in Oryza sativa [11], 400 in Populus trichocarpa [12], 333 in Medicago truncatula [13], 54 in Carica papaya [14], 534 in Vitis vinifera [15] and 158 in Lotus japonicas [16] have been identified. Earlier genome-wide studies have demonstrated that TNL subfamily is abundant in dicots while absent in cereals (monocots) [17]. The presence of the full length of TNL and CNL types in the common ancestor (mosses) of both angiosperms and gymnosperms and exceptional presence of truncated domains of TN or TX type proteins in cereals indicate that the TNL class might have been lost in monocot plants $[9,18]$. On the chromosomes, the NBS-LRR R genes are arranged in clusters. The genes in the clusters could be homogenous (often tandem duplicated from single ancestor gene) or heterogenous (with different protein domains) [19-21]. However, the variation of the number and sequences of the $\mathrm{R}$ genes presented in the Brassica lineage since split from the Arabidopsis lineage and their distributions in chromosomes are unknown.

The genera Brassica and Arabidopsis, both belong to the mustard family Brassicaceae (Cruciferae), are a model plant and a model crop, respectively. The two genera shared a latest and obviously detectable alpha genome duplication event before their divergence $\sim 20$ million years ago (MYA) and subsequently Brassica ancestor underwent a whole genome triplication event (common to the tribe Brassicaceae) 16 MYA [22-25]. In Brassica, interspecific cytogenetic relationship between important crops (oilseed and vegetables) is well-described by a " $U$ " triangle where each two diploid species [B.rapa (AA, $2 n=20)$, B. oleracea $(C C, 2 n=18)$ and B. nigra $(B B$, $2 \mathrm{n}=16$ )] formed a tetraploidy species [B.napus (AACC, $2 \mathrm{n}=38$ ), B. juncea (AABB, $2 \mathrm{n}=36$ ) or B. carinata (BBCC, $2 \mathrm{n}=34$ )] [26]. This well-established phylogenetic relationship provides a chance to trace evolution of the R genes between wild plants and their relative crops. The present study is to identify $\mathrm{R}$ genes on genome-wide scale in $B$. oleracea and B. rapa and provide insights into their evolutionary history and disease resistance.

\section{Methods}

\section{Data resource}

Arabidopsis thaliana, Brassica rapa and Brassica oleracea genomic and annotation data was downloaded from the TAIR10 (http://www.arabidopsis.org) [27], the BRAD database (http://brassicadb.org/brad/) [28] and the Bolbase database (http://ocri-genomics.org/bolbase) [29], respectively. Theobroma cacao genomic data was downloaded from http://cocoagendb.cirad.fr/, Populus trichocarpa genomic 
data was downloaded from JGI database (http://www.phytozome.net/poplar.php), Vitis vinifera genomic data was downloaded from http://www.genoscope.cns.fr/externe/GenomeBrowser/Vitis/, Medicago truncatula genomic data was downloaded from http://www.medicago.org/. The Hidden Markov Model (HMM) profiles of NBS and TIR domain (PF00931 and PF01582) were retrieved from Pfam 26.0 (http://Pfam.sanger.ac.uk) [30]. B. rapa and B. oleracea illumina RNA-seq data were obtained from the Gene Expression Omnibus (GEO) database with accession numbers GSE43245 and GSE42891 respectively.

\section{Identification of $B$. oleracea genes that encode NBS domain and NBS-associated conserved domains}

In the draft genome of $B$. oleracea, NBS-encoding genes were identified through Hidden Markov Model (HMM) profile corresponding to the Pfam NBS (NB-ARC) family PF00931 domain using HMMER V3.0 programme with "trusted cutoff" as threshold [31]. From the selected protein sequences screened through NBS domain, high quality sequences were aligned through CLUSTALW [32] and used to construct $B$. oleracea specific NBS profile using the "hmmbuild" module by HMMER V3.0 programme. With this model final set of NBS-encoding proteins were identified and only 157 proteins were selected as NBS candidate genes with stringent parameters. The NBS R-gene family is subdivided into different groups based on the structure of the $\mathrm{N}$-terminal and $\mathrm{C}$-terminal domains of the protein. For the identification of $\mathrm{N}$-terminal and $\mathrm{C}$-terminal domains of NBS-encoding genes, we used HMMPfam and HMMSmart for detection. We further employed PAIRCOIL2 [33] (P score cut-off of 0.025) and MARCOIL [34] programs with a threshold probability of 90 to confirm Coiled-Coil (CC) motif. From the result generated by these programs, we selected overlapping sequences as candidate genes with CC motif. We used same procedures to identify genes that contain TIR domain only and excluded the NBS-encoding genes as TIR-X genes. NBS-encoding genes in A. thaliana and $B$. rapa have been reported earlier but in order to get the latest NBS-encoding genes in these two species for our comparative analysis, we followed the same procedures to screen NBS candidate genes in B. rapa and A. thaliana for consistency.

\section{Assigning the location of NBS-encoding genes to $B$. oleracea and $B$. rapa genome}

The physical position of NBS-encoding genes was mapped to the 9 and 10 pseudo-molecular chromosomes of B. oleracea and B. rapa using GFF file which was downloaded from Bolbase [29] and BRAD [28] database respectively. After that, we used in-house perl script to draw graphic potryl of NBS-encoding genes on pseudomolecular chromosomes with SVG module [35].

\section{Identification of tandem duplicated arrays}

To detect the generated mechanism of NBS-encoding genes, BLASTP program [36] was employed to identify the tandem duplicated genes using protein sequences with E-value cutoff $\leq 1 \mathrm{e}-20$, and one unrelated gene was allowed within a tandem array.

\section{Alignment and phylogenetic analysis of NBS-encoding genes}

According to location of conserved domains for NBS (Nucleotide-binding Site) in complete predicted NBS protein sequences, conserved domain sequences of NBS-encoding genes were extracted and aligned using the programme Clustal W [32] with default options for the phylogenetic analysis among 3 species. The poor alignment sequences were excluded by manually curation using Jalview [37]. The resulting sequences were used to construct a phylogenetic tree using Maximum Likelihood (ML) method in MEGA 5.0 [38] with 1000 replications.

\section{Orthologous gene pairs between $B$. rapa, $A$. thaliana and B. oleracea}

Orthologous gene pairs provide information about the evolutionary relationship between different species. In our study, we used two steps to detect gene pairs precisely. First, MCscan programme [39] was employed to identify orthologous regions with the parameters $(e=1 \mathrm{e}-20, \mathrm{u}=1$ and $\mathrm{s}=5$. Parameter of $\mathrm{s}=5$ ) between $B$. rapa, A. thaliana and $B$. oleracea genomes. Second, after extracting orthologous regions that contained NBS-encoding genes, orthologous gene pairs of NBS-encoding genes were extracted.

Non-synonymous/synonymous substitution (Ka/Ks) ratios of gene pairs between B. rapa, A. thaliana and B. oleracea For the estimation of selection mode for the NBS-encoding genes among $B$. oleracea, B. rapa and $A$. thaliana, the ratio of the rates of nonsynonymous to synonymous substitutions $(\mathrm{Ka} / \mathrm{Ks})$ of all orthologous gene pairs were calculated for each branch of the phylogenetic tree using PAML software [40]. For each subtree of NBS orthologous gene pairs among 3 species, model 1 with a free $\mathrm{Ka} / \mathrm{Ks}$ ratio was calculated separately for each branch. The $\mathrm{Ka} / \mathrm{Ks}$ values associated with terminal branches between modern species and their most recent reconstructed ancestors were employed in the subsequent analyses. In order to detect selection pressure, $\mathrm{Ka} / \mathrm{Ks}$ ratio greater than 1 , less than 1 and equal to 1 represents positive selection, negative or stabilizing selection and neutral selection, respectively.

\section{RNA-seq data analysis of NBS-encoding genes}

For expression profiling of NBS-encoding genes, we used RNA-seq data that was generated earlier and submitted into GEO database. Transcript abundance is calculated by fragments per kilobase of exon model per million mapped 
reads (FPKM) and the FPKM values were $\log 2$ transformed. A hierarchical cluster was created using the Cluster 3.0 and heat map generated using TreeView Version 1.60 software [41].

\section{Results}

Identification and classification of NBS genes in A. thaliana and Brassica species

Although, previously NBS-encoding $\mathrm{R}$ genes in $A$. thaliana and $B$. rapa were described by Meyers et al. [10] and Mun et al. [42] respectively, but their analysis were based on old version of TAIR in $A$. thaliana and incomplete genome sequences in $B$. rapa. In the genome assemblies of B. oleracea, B. rapa and A. thaliana, 157, 206 and 167 NBS-encoding genes respectively were identified using the HMM profile from the Pfam database [30]. According to gene structure and protein motifs, we categorized these putative NBS-encoding genes into seven different classes: TNL (40, 93 and 79 for B. oleracea, B. rapa and $A$. thaliana, respectively), TIR-NBS (29, 23 and 17), CNL (6, 19 and 17), CC-NBS (5, 15 and 8), NBS-LRR (24, 27 and 20) and NBS (53, 29 and 26) (Table 1, Additional file 1: Table S1). We employed HMM search to identify genes with open reading frames that encode TIR domain based on whole genomes of sequenced plant species. By excluding genes that contain NBS domains, we obtained the genes that encode only TIR domain (TIR-X type genes). Although, the number of NBS-encoding genes in $B$. oleracea is less than that of $A$. thaliana and $B$. rapa but genes with truncated domains of NBS, TIR-NBS and TIR-X are more than these species. The total number of NBS-encoding genes in these three species is very close regardless of genome size and WGD/WGT, suggesting WGT might not result in more $\mathrm{R}$ genes in Brassica species. Much

Table 1 Statistics of predicted NBS-encoding genes in sequenced plant species

\begin{tabular}{lllllllll}
\hline \multicolumn{2}{c}{ Categories } & Bo & Br & At & Tc & Pt & Vv & Mt \\
\hline NBS-LRR type & TIR-NBS-LRR & 40 & 93 & 79 & 8 & 78 & 97 & 118 \\
& CC-NBS-LRR & 6 & 19 & 17 & 82 & 120 & 203 & 152 \\
& NBS-LRR & 24 & 27 & 20 & 104 & 132 & 159 & - \\
NBS type & TIR-NBS & 29 & 23 & 17 & 4 & 10 & 14 & 38 \\
& CC-NBS & 5 & 15 & 8 & 46 & 14 & 26 & 25 \\
NBS & 53 & 29 & 26 & 53 & 62 & 36 & 328 \\
Total NBS & & 157 & 206 & 167 & 297 & 416 & 535 & 661 \\
Total TIR-NBS & 69 & 116 & 96 & 12 & 88 & 111 & 156 \\
Total CC-NBS & 11 & 34 & 25 & 128 & 134 & 229 & 177 \\
TIR-X* & 82 & 42 & 46 & 17 & 67 & 10 & 92 \\
Total & 239 & 248 & 213 & 314 & 483 & 545 & 753 \\
\hline
\end{tabular}

Note: Bo-B. oleracea; Br-B. rapa; At-A. thaliana; Tc-T. cacao, Pt-P. trichocarpa, $V v$-V. vinifera; $M t-M$. truncatula

*identified in present study. more TNL type genes than CNL ones, and more TIR-NBS than CC-NBS were also observed in these three species.

\section{Genomic distribution on chromosomes/pseudomolecular chromosomes}

NBS-encoding genes for the three species were mapped onto pseudo-molecules/ chromosomes [121 (77.1\%) genes in B. oleracea, 197 (95.6\%) genes in B. rapa and 167 (100\%) genes in $A$. thaliana] and the rest [36 (22.9\%) genes in $B$. oleracea and 9 (4.4\%) genes in B. rapa] were located on the unanchored scaffolds (Figure 1). The distribution of these genes is uneven: some chromosomes (e. g. C07 in B. oleracea representing the $20.7 \%$ of the NBS-encoding genes) have more genes and the rest chromosomes have fewer genes (e. g. C05 in B. oleracea), and many of these genes reside in a cluster manner. $\mathrm{R}$ genes existing in clusters may facilitate the evolutionary process through producing novel resistance genes via genome duplication, tandem duplication and gene recombination [43]. According to the cluster defined by Richly et al. [44] and Meyers et al. [10] as two or more genes falling within eight ORFs, we found that the percentage of NBS genes on chromosomes in clusters in $B$. oleracea $(60.3 \%)$ and $A$. thaliana $(61.7 \%)$ is higher than that of $B$. rapa (59.4\%). In B. oleracea, 73 NBS genes, representing $60.3 \%$ of total genes on chromosomes, were located in 24 clusters and the remaining 48 genes were singletons. Five clusters containing 19 NBS genes were identified on the chromosome $\mathrm{C} 07$ (Figure $1 \mathrm{~A}$ ). The $B$. rapa genome carries 117 (59.4\%) NBS genes with TIR domain and CC motif in 43 clusters and remaining 80 genes were found as singletons on chromosomes. Among the 43 clusters, 11 with 31 genes were located on chromosome A09 (Figure 1B). In A. thaliana, 103 (61.7\%) NBS genes with TIR domain and CC motif were mapped in 37 clusters whereas the remaining 64 genes were found as singletons. The numbers of genes in clusters ranged from two to six in both Brassica species and two to nine in A. thaliana.

Further, more numbers of homogenous clusters was observed in B. rapa and A. thaliana than B. oleracea. In $B$. oleracea among 24 identified clusters, 5 were homogenous and one of them containing four genes (Bol040038, Bol040039, Bol040042, and Bol040045) with TN domain configuration was located on chromosome C06. Most of the clusters (18) are heterogenous with distantly related NBS domains. Fifteen clusters in each of B. rapa and $A$. thaliana were found to be homogenous containing the NBSencoding genes mostly from TNL domain combination.

\section{Phylogenetic analysis of NBS-encoding genes in B. oleracea,}

\section{$B$. rapa and $A$. thaliana}

Comparative phylogenetic relationship of NBS-encoding genes in B. oleracea, $A$. thaliana and $B$. rapa represents two major groups of TNL (348 genes) and CNL (138 genes) containing genes from three species. In composite 
(See figure on previous page.)

Figure 1 NBS-encoding genes and corresponding clusters distribution of NBS-encoding genes in $B$. rapa and $B$. oleracea genomes.

A. A01 A10 represent pseudo-chromosomes of B. rapa genome. B. C01 C09 represent pseudo-chromosomes of B. oleracea genome. Green bars represent pseudo-chromosomes. Black line on green bars stands for the location of NBS-encoding genes on pseudo-chromosomes. Colorful boxes stand for clusters of NBS-encoding genes in corresponding genomes.

phylogenetic tree, TNL and CNL groups were further divided into three sub-groups, TNL-I-III and CNL-I-III (Additional file 2: Figure S1). We did not observe any strict grouping of $\mathrm{N}, \mathrm{NN}$ and $\mathrm{NL}$ domain containing proteins and these kinds of proteins were clustered in both TNL and CNL groups. From phylogenetic tree, we can differentiate that the number of NBS-encoding genes for three species in each subgroup was not identical. In TNL group all sub-trees comprised genes with full length TIR-NBSLRR ORFs, truncated and complex domains. TNL-I subgroup was found to be the largest one containing 245 NBS members in total and greater part in this subgroup was from B. rapa (106 NBS members). This subgroup included the largest part of the full length TNLs and second and third prevalent classes are $\mathrm{TN}$ and $\mathrm{N}$ type genes respectively. The domain arrangement was found to be highly diverse and NBS-encoding genes from three species with thirteen different complex and unusual domain combinations of TNNL, TCNL, TNTN, TNLT, TNNTNNL, NLTNL, NNL, TNLTNL, CTN, TNN, TTN, TNLN and LTNL were identified in this subgroup. In subgroup TNL-II, more than half of the genes were from B. oleracea and others were from B.rapa and A. thaliana. This subgroup along with various complex domain arrangement containing genes also carried most of the full length TNLs. TNL-III was the smallest subgroup with majority of genes from $B$. oleracea (5 genes) and a single gene from each of $\mathrm{B}$. rapa and $A$. thaliana. B. oleracea gene, Bol044437 with unusual domain arrangement TNNL also clustered in this subgroup.

$\mathrm{CNL}$ group was further divided into three distinct subgroups represented by genes from all the three species and we also observed one CNL subgroup which was already recognized in $A$. thaliana. However, CNL group is not much variant and only few complex domain arrangements are evident; NNL, CNNL and CNNN. In CNL-1 subgroup, out of 5 clustered $A$. thaliana genes, 4 genes (AT4G33300.1, AT1G33560.1, AT5G04720.1 and AT5G47280.1) were also grouped in the respective $A$. thaliana CNL-A subgroup as identified and described by Meyers et al. 2003. Both CNL-II and CNL-III subgroups included most of NBS-encoding genes from $B$. rapa and $A$. thaliana and fewer genes from $B$. oleracea species. NBS-encoding genes with $\mathrm{N}$ and $\mathrm{CN}$ type truncated domains were observed more in CNL-II subgroup and one $B$. rapa gene (Bra037453) with unusual domain, CNNN also clustered here. Subgroup CNL-III was represented by 73 genes and most of the members (36) were full length CNL ORFs. Four B. rapa genes (Bra030779, Bra027097, Bra019752, Bra015597) with unusual domains NNL and CNNL were also identified in this subgroup.

\section{Expression analysis of NBS-encoding genes in different tissues}

To investigate the expression pattern of NBS-encoding genes, we compared the transcript abundance in different tissues using RNA-seq data from GEO database. The expression profile of NBS-encoding genes in $B$. oleracea could be classified into two major groups (Bol-A and BolB) (Additional file 3: Figure S2A). Eighty eight genes belonging to Group Bol-A, further divided into two subgroups, Bol-A1 and Bol-A2. In B. oleracea in subgroup Bol-A1, three genes (Bol017532, Bol029866 and Bol013571) expressed relatively higher in root and stalk indicating their tissue-specific role in these tissues. Majority of genes in subgroup Bol-A2 were found to be upregulated in root and callus (for example, Bol038522 displayed more expression in root and callus and Bol024369 was abundant only in root tissue) but down regulated in stalk, leaf, flower and silique. Up regulation of these genes in callus suggests their induction under wounding. However, eighteen genes in group Bol-B displayed differential expression in different tissues and among all the genes in this subgroup, Bol009890 exhibited highest expression in leaf and Bol 036980 showed more transcript level in flower tissue.

In $B$. rapa, genes could be categorized into two main groups, Bra-A and Bra-B (Additional file 3: Figure S2B). The Bra-A group was further classified into Bra-A1 (74 genes), Bra-A2 (45 genes) and Bra-A3 (28 genes). In subgroup Bra-A1 of B. rapa, most of genes displayed high transcript accumulation in root, stalk and callus which indicates that they may expression pattern differentially. Among the other genes, Bra006146 showed high expression in vegetative tissue (root, stalk and leaf) and Bra004192 and Bra035103 highly expressed in stalk and leaf. In subgroup Bra-A2, where a number of genes were expressed more in root and callus. However, Bra018810 displayed highest expression in silique suggesting its silique-specific role. In Subgroup Bra-A3, some genes showed the preferential transcript level in stalk and flower and some genes relatively expressed higher in flower, silique and callus. For example, Bra008055 accumulated more transcripts in leaf, flower and callus, Bra008056 in flower and Bra026094 in stalk and silique. Most of genes in group Bra-B showed high expression in stalk and leaf as compared to other tissues and Bra009882, Bra008053, 
Bra018834, Bra027866, Bra026368 and Bra030778 highly expressed in leaf tissues. This may specify that genes in this subgroup act as positive regulator in leaf tissues.

Taken together, we suggest that NBS-encoding genes exhibited differential expression pattern in different tissues and several genes are induced by wounding in $B$. oleracea and B. rapa genomes. Some NBS-encoding genes showed higher expression in same tissue indicating their functional conservation, but others were more abundant in different tissues which point toward their functional differences. According to expression pattern of NBS-encoding genes in different tissues, it would be interesting to functionally characterize these genes for pathogen defense response, especially race- and speciesspecific pathogens in Brassica species.

\section{Whole genome duplication analysis of NBS-encoding genes}

A. thaliana genome has experienced two recent whole genome duplication (named $\alpha$ and $\beta$ ) within the crucifer
(Brassicaceae) lineage and one triplication event $(\gamma)$ that is probably shared by most dicots (asterids and rosids) [45]. The ancestor of diploid Brassica species and A. thaliana lineages diverged about 20 MYA and subsequently a whole genome triplication (WGT) event occurred in the Brassica ancestor approximately 16 MYA. As WGT of the Brassica ancestor, NBS-encoding genes in the A. thaliana genome might have triplicated orthologous copies in B. rapa and B. oleracea. Since, $A$. thaliana is considered a model plant system for plant molecular biology research and most of its genes have been functionally characterized. Therefore, we traced these orthologous gene pairs between A. thaliana and Brassica species to detect the NBS-encoding genes in evolutionary history. From analysis of orthologous regions for genome-wide comparative analysis, we obtained 42 orthologous gene pairs between $A$. thaliana and B. oleracea, 62 between A. thaliana and $B$. rapa and 24 between $B$. oleracea and $B$. rapa, which are shown in Figure 2 developed by Circos software [46] (Figure 2).

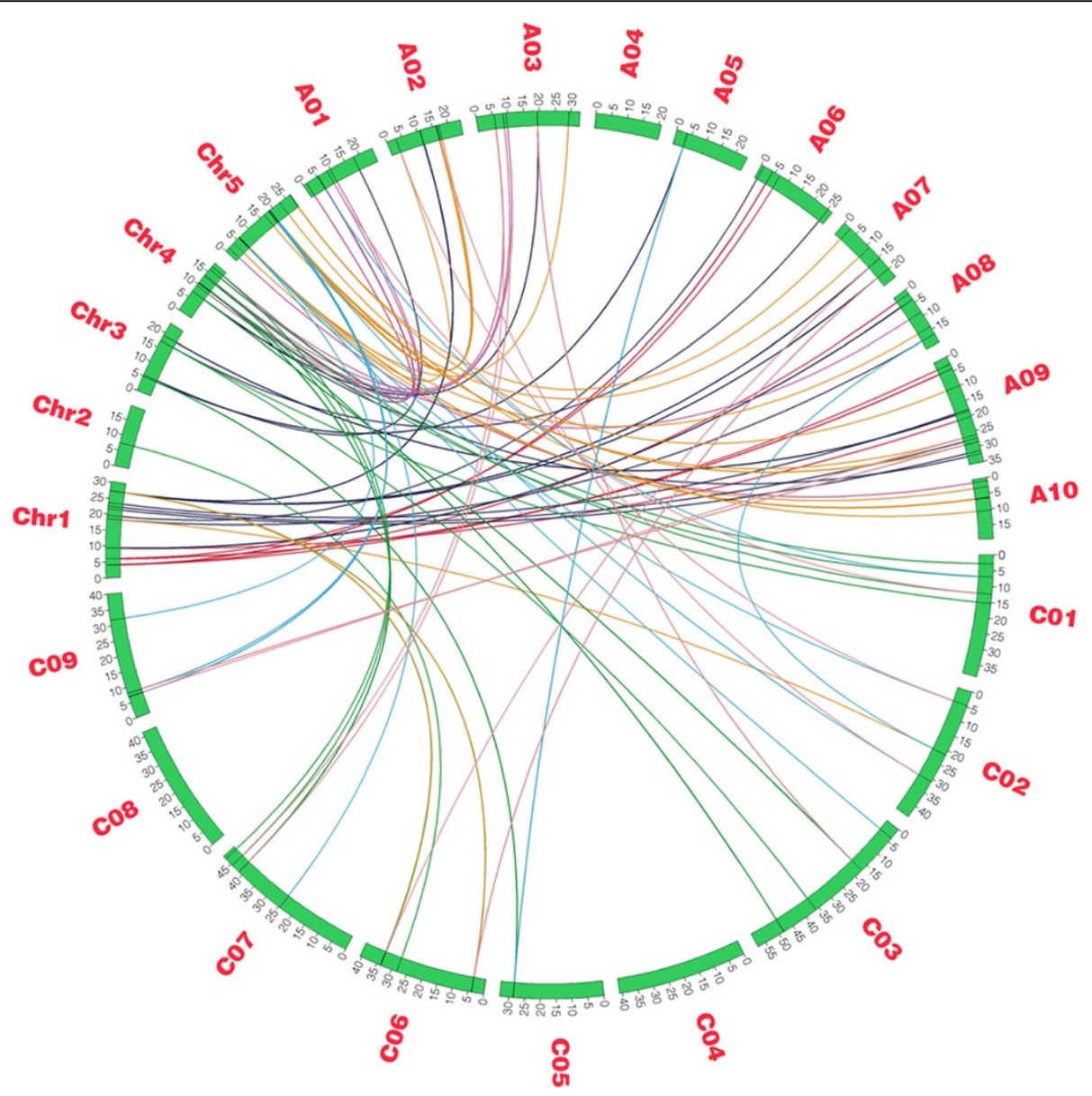

Figure 2 Syntenic relationship of NBS-encoding genes between A. thaliana and Brassica genomes. Green bars represent chromosomes of three species. A01 A10 represent pseudo-chromosomes of B. rapa genome, C01 C09 represent pseudo-chromosomes of B. oleracea genome and Chr $1 \sim$ Chr5 represent chromosomes of $A$. thaliana genome. Black line on green bars stands for the location of NBS-encoding genes on chromosomes/pseudo-chromosomes. Colorful lines stand for the relationship of orthologous gene pairs between different species. 
Out of 42 gene pairs between $A$. thaliana and B. oleracea, 26 A thaliana NBS genes were shown to retain one copy, 5 A. thaliana NBS genes retained two copies and only 2 genes corresponding to AT4G19500.1 and AT4G19510.1 each preserved tripled copies after triplication in $B$. oleracea. In total, 42 NBS genes in B. oleracea genome have 33 corresponding genes in $A$. thaliana genome. A. thaliana corresponding genes in $B$. oleracea were located on different chromosomes and some gene pairs (which retained single copy in B. oleracea) and 3 out of 5 A. thaliana corresponding genes (which retained two copies in $B$. oleracea) preserved domain structure (Table 2).

Out of 62 gene pairs between $A$. thaliana and B. rapa, 40 A thaliana NBS genes were shown to retain one copy, 8 A. thaliana NBS genes retained two copies and only two genes (AT4G26090.1 and AT1G72890.1) preserved tripled copies in B. rapa. At last, we got $50 \mathrm{~A}$. thaliana NBS genes compared to $62 \mathrm{NBS}$ genes in $B$. rapa genome. Gene pairs in $B$. rapa corresponding to $A$. thaliana were located on different chromosomes. Further, some genes (which retained single copy in B. rapa), 5 out of 8 A. thaliana NBS genes (which retained two copies in $B$. rapa) and 2 genes (which retained tripled copies in B. rapa) preserved domain configuration in $B$. rapa (Table 3 ).

The ancestor of Brassica species has experienced whole genome triplication and thus provided sufficient genomic materials to study retention and loss of NBS-encoding genes. In order to detect retention or loss of NBSencoding genes after WGT, we studied the A. thaliana NBS genes, which have corresponding genes in Brassica species. There are 33 A. thaliana NBS genes compared to 42 B. oleracea NBS genes and 50 A. thaliana NBS genes compared to $62 \mathrm{~B}$. rapa NBS genes, which have 24 overlapping NBS genes. In other words, 59 NBS genes in A. thaliana genome were identified on triplicated regions and generated triple copies in Brassica species, representing $35.32 \%$ of total NBS genes in A thaliana genome. Because of evolutionary constraints, 42 NBS genes were retained on triplicated regions, representing $26.75 \%$ of total NBS genes in B. oleracea genome and 62 NBS genes were retained on triplicated blocks, which represent $30.1 \%$ of whole NBS genes in B. rapa genome.

\section{Tandem duplication analysis of NBS-encoding genes}

Whole genome and/or tandem duplication is thought to be source of complexity and diversity for plant species and allow them to adapt to the changed environmental conditions. In B. oleracea genome, 68 of 157 identified NBSencoding genes, representing $43.3 \%$ genes were formed by tandem duplication and distributed in 26 tandem arrays of 2-6 genes. The chromosome map identified 21 tandem arrays including 57 NBS-encoding genes unevenly distributed on seven of the nine chromosomes and remaining 11 genes were unanchored on scaffold sequences. Genes with
CNL or CN domain were not appeared in tandem arrays. Single tandem duplicated array containing two genes were identified on each of chromosome C01 and C05 with $\mathrm{N}$ and NL domains. Each of the chromosomes C02 and C03 carried four tandem arrays with 2-4 genes. The chromosome C06 (2-5 genes in arrays) and C09 (2-4 genes in arrays) carried two and three tandem arrays respectively. The highest number of tandem arrays (6) with 17 genes was found on chromosome $\mathrm{C07}$ which contains the highest number of $\mathrm{R}$ genes in the genome. In $A$. thaliana genome, out of 167 NBS genes 93 (55.7\%) genes were tandemly duplicated and positioned on chromosomes in 37 tandem arrays. The tandem duplicated genes were distributed in tandem arrays of 2-6 genes. In B. rapa genome, 97 genes (47.1\%) were tandemly duplicated and 93 genes were located on chromosomes in 38 tandem arrays while two tandem arrays were located on scaffold sequences. The number of duplicated genes range from 2-5 genes in tandem arrays (Table 4, Additional file 4: Table S2).

In order to detect the fate of tandem arrays in Brassica lineage after split from Arabidopsis thaliana, we investigated the orthologous gene pairs in tandem array among $B$. oleracea, B. rapa and $A$. thaliana genomes. 10 twogene tandem arrays of $A$. thaliana have corresponding two-gene tandem arrays in $B$. oleracea and $B$. rapa genomes, and further 7 and 9 two-gene tandem arrays have retained their copies in B. rapa and B. oleracea genome, respectively (Additional file 5: Table S3). Out of 10 twogene tandem arrays in $A$. thaliana, 4 A thaliana two-gene tandem arrays were co-retained tandem arrays and have corresponding two-gene tandem arrays in $B$. rapa and $B$. oleracea genome, 3 two-gene tandem arrays have retained in $B$. rapa genome and 3 two-gene tandem arrays have retained in B. oleracea genome. Among 157 NBSencoding genes in B. oleracea, 68 genes were tandem duplicated genes. 18 of 68 genes were conserved and have ancient copies, indicating that those 18 genes were generated before divergence of $A$. thaliana and Brassica ancestor. Consequently, 50 NBS-encoding genes were distributed in species-specific tandem arrays in B. oleracea genome. In $B$. rapa genome, 97 tandem duplicated genes representing $47.1 \%$ of 206 NBS-encoding genes in total, contained 14 genes belonging to tandem of pre-split. 83 genes were species-specific tandem duplicated genes in $B$. rapa genome. There are 93 genes identified as tandem duplicated genes in A. thaliana genome and 20 tandem duplicated genes are pre-split tandem genes, named common tandem duplicated genes, which were generated before divergence of $A$. thaliana and Brassica ancestor. Out of 20 common tandem genes, 8 genes retained copies in Brassica species and those corresponding co-retained tandem genes were race-specific tandem duplicated genes in Brassica species. 
Table 2 Orthologous gene pairs of NBS-encoding genes between $A$. thaliana and $B$. oleracea genomes

\begin{tabular}{|c|c|c|c|c|c|c|c|c|c|}
\hline \multirow[t]{2}{*}{ A. thaliana } & \multicolumn{4}{|c|}{ NBS-encoding genes in A. thaliana } & \multirow[t]{2}{*}{ B. oleracea } & \multicolumn{4}{|c|}{ NBS-encoding genes in $B$. oleracea } \\
\hline & Gene_Type & Location & ORF Length & No. of exons & & Gene_Type & Location & ORF Length & No. of exons \\
\hline AT1G27170.1 & TIR-NBS-LRR & Chr1 & 4,858 & 5 & Bol037684 & TIR-NBS-LRR & NY & 4,783 & 5 \\
\hline AT1G50180.1 & NBS & Chr1 & 2,901 & 5 & Bol011780 & CC-NBS & $\mathrm{CO2}$ & 3,507 & 3 \\
\hline AT1G63730.1 & TIR-NBS-LRR & Chr1 & 3,362 & 4 & Bol022619 & NBS-LRR & NY & 4,656 & 3 \\
\hline AT1G72870.1 & TIR-NBS & Chr1 & 2,161 & 2 & Bol026308 & TIR-NBS & $\mathrm{CO6}$ & 2,317 & 2 \\
\hline \multirow[t]{2}{*}{ AT1G72890.1 } & TIR-NBS & Chr1 & 1,770 & 2 & Bol026304 & NBS & C06 & 830 & 2 \\
\hline & & & & & Bol040038 & TIR-NBS & C06 & 1,565 & 3 \\
\hline \multirow[t]{2}{*}{ AT1G72950.1 } & TIR-NBS & Chr1 & 1,395 & 2 & Bol026303 & TIR-NBS & C06 & 1,232 & 2 \\
\hline & & & & & Bol040042 & TIR-NBS & C06 & 2,357 & 3 \\
\hline AT2G17060.1 & TIR-NBS-LRR & Chr2 & 4,466 & 6 & Bol023868 & CC-NBS-LRR & C06 & 2,938 & 2 \\
\hline AT3G14460.1 & NBS-LRR & Chr3 & 4,274 & 1 & Bol005097 & NBS-LRR & C05 & 3,623 & 1 \\
\hline AT3G14470.1 & NBS-LRR & Chr3 & 3,307 & 1 & Bol005098 & NBS & C05 & 9,997 & 3 \\
\hline \multirow[t]{2}{*}{ AT3G46730.1 } & NBS & Chr3 & 2,543 & 1 & Bol041411 & NBS & $\mathrm{CO3}$ & 164 & 1 \\
\hline & & & & & Bol018762 & NBS & C01 & 869 & 1 \\
\hline AT3G51560.1 & TIR-NBS-LRR & Chr3 & 4,105 & 5 & Bol010610 & TIR-NBS-LRR & NY & 5,368 & 4 \\
\hline AT3G51570.1 & TIR-NBS-LRR & Chr3 & 4,098 & 5 & Bol010611 & TIR-NBS & NY & 4,246 & 6 \\
\hline \multirow[t]{2}{*}{ AT4G12010.1 } & TIR-NBS-LRR & Chr4 & 4,182 & 5 & Bol008302 & TIR-NBS-LRR & NY & 5,143 & 5 \\
\hline & & & & & Bol030522 & TIR-NBS-LRR & $\mathrm{CO3}$ & 3,222 & 4 \\
\hline AT4G12020.2 & NBS-LRR & Chr4 & 7,992 & 16 & Bol030521 & NBS & $\mathrm{CO3}$ & 4,504 & 6 \\
\hline AT4G19050.1 & NBS-LRR & Chr4 & 3,684 & 2 & Bol009352 & NBS-LRR & C01 & 3,341 & 1 \\
\hline \multirow[t]{3}{*}{ AT4G19500.1 } & TIR-NBS-TIR-NBS-LRR & Chr4 & 4,736 & 5 & Bol003710 & NBS-LRR & NY & 4,676 & 3 \\
\hline & & & & & Bol024375 & TIR-NBS-LRR & $\mathrm{CO}$ & 4,087 & 6 \\
\hline & & & & & Bol029862 & TIR-NBS-LRR & $\mathrm{CO3}$ & 2,445 & 4 \\
\hline \multirow[t]{3}{*}{ AT4G19510.1 } & TIR-NBS-LRR & Chr4 & 5,316 & 6 & Bol003711 & TIR-NBS & NY & 3,941 & 4 \\
\hline & & & & & Bol024376 & NBS & $\mathrm{CO}$ & 3,424 & 4 \\
\hline & & & & & Bol029861 & TIR-NBS & $\mathrm{CO3}$ & 3,947 & 5 \\
\hline AT4G19520.1 & TIR-NBS-LRR & Chr4 & 4,421 & 4 & Bol024371 & TIR-NBS-LRR & $\mathrm{CO}$ & 13,901 & 6 \\
\hline AT4G19530.1 & TIR-CC-NBS-LRR & Chr4 & 5,538 & 5 & Bol024372 & TIR-NBS-LRR & $\mathrm{CO}$ & 6,114 & 4 \\
\hline AT4G26090.1 & CC-NBS-LRR & Chr4 & 3,534 & 1 & Bol039594 & CC-NBS-LRR & C01 & 2,723 & 1 \\
\hline AT4G27190.1 & CC-NBS-LRR & Chr4 & 2,957 & 1 & Bol042325 & CC-NBS-LRR & $\mathrm{CO}$ & 3,053 & 1 \\
\hline AT4G33300.1 & NBS-LRR & Chr4 & 5,475 & 5 & Bol013568 & NBS & C01 & 2,067 & 5 \\
\hline AT4G36140.1 & TIR-NBS-TIR-NBS-LRR & Chr4 & 5,523 & 7 & Bol018676 & NBS-LRR & $\mathrm{CO}$ & 4,440 & 7 \\
\hline AT5G04720.1 & NBS & Chr5 & 3,172 & 5 & Bol002454 & NBS-LRR & NY & 3,733 & 5 \\
\hline \multirow[t]{2}{*}{ AT5G17880.1 } & TIR-NBS-LRR & Chr5 & 4,225 & 6 & Bol019768 & TIR-NBS & C09 & 2,856 & 5 \\
\hline & & & & & Bol034463 & TIR-NBS-LRR & $\mathrm{CO3}$ & 2,203 & 3 \\
\hline AT5G17970.1 & TIR-NBS-LRR & Chr5 & 2,620 & 4 & Bol021382 & NBS-NBS & $\mathrm{CO} 2$ & 4,845 & 2 \\
\hline AT5G45200.1 & TIR-NBS-LRR & Chr5 & 6,365 & 5 & Bol032050 & TIR-NBS-LRR & C09 & 9,525 & 4 \\
\hline AT5G45210.1 & TIR-NBS-LRR & Chr5 & 2,913 & 4 & Bol032051 & TIR-NBS & CO9 & 5,857 & 4 \\
\hline AT5G45240.1 & TIR-NBS-LRR & Chr5 & 5,383 & 10 & Bol005623 & NBS & C07 & 2,409 & 3 \\
\hline AT5G45250.1 & TIR-NBS-LRR & Chr5 & 4,108 & 5 & Bol032054 & TIR-NBS & C09 & 4,575 & 6 \\
\hline AT5G45490.1 & NBS & Chr5 & 1,394 & 1 & Bol022842 & NBS & $\mathrm{CO2}$ & 728 & 1 \\
\hline AT5G46450.1 & TIR-NBS-LRR & Chr5 & 3,928 & 5 & Bol032126 & NBS-LRR & C09 & 2,893 & 3 \\
\hline AT5G46470.1 & TIR-NBS-LRR & Chr5 & 7,040 & 6 & Bol032125 & NBS & CO9 & 671 & 2 \\
\hline
\end{tabular}


Table 3 Orthologous gene pairs of NBS-encoding genes between $A$. thaliana and $B$. rapa genomes

\begin{tabular}{|c|c|c|c|c|c|c|c|c|c|}
\hline \multirow[t]{2}{*}{ A. thaliana } & \multicolumn{4}{|c|}{ Attribute of NBS-encoding genes in A. thaliana } & \multirow[t]{2}{*}{ B. rapa } & \multicolumn{4}{|c|}{ Attribute of NBS-encoding genes in B. rapa } \\
\hline & Gene_Type & Location & ORF_Length & No. of exons & & Gene_Type & Location & ORF_Length & No. of exons \\
\hline AT1G50180.1 & NBS & Chr1 & 2,901 & 5 & Bra014241 & CC-NBS & A08 & 4,047 & 3 \\
\hline AT1G58410.1 & NBS & Chr1 & 3,070 & 3 & Bra027866 & CC-NBS & A09 & 2,986 & 3 \\
\hline \multirow[t]{2}{*}{ AT1G59620.1 } & NBS & Chr1 & 3,401 & 5 & Bra035424 & CC-NBS & NY & 3,017 & 3 \\
\hline & & & & & Bra016781 & CC-NBS-LRR & A08 & 2,662 & 2 \\
\hline AT1G12290.1 & CC-NBS-LRR & Chr1 & 2,888 & 1 & Bra026979 & CC-NBS-LRR & A09 & 2,744 & 1 \\
\hline \multirow[t]{3}{*}{ AT4G26090.1 } & CC-NBS-LRR & Chr4 & 3,534 & 1 & Bra013947 & CC-NBS-LRR & $\mathrm{A} 01$ & 2,723 & 1 \\
\hline & & & & & Bra019063 & CC-NBS-LRR & A03 & 3,029 & 1 \\
\hline & & & & & Bra037139 & CC-NBS-LRR & A09 & 3,023 & 4 \\
\hline AT3G51560.1 & TIR-NBS-LRR & Chr3 & 4,105 & 5 & Bra036791 & TIR-NBS-LRR & A09 & 10,379 & 6 \\
\hline AT1G17610.1 & NBS & Chr1 & 1,462 & 1 & Bra030997 & NBS & A09 & 1,262 & 1 \\
\hline AT1G52660.1 & NBS & Chr1 & 1,321 & 3 & Bra018980 & NBS & A06 & 1,308 & 3 \\
\hline AT3G15700.1 & NBS & Chr3 & 1,240 & 2 & Bra021130 & NBS & $\mathrm{A} 01$ & 1,322 & 2 \\
\hline AT3G46710.1 & NBS & Chr3 & 2,543 & 1 & Bra018198 & NBS & A06 & 2,078 & 5 \\
\hline AT4G19060.1 & NBS & Chr4 & 1,384 & 1 & Bra013373 & NBS & $\mathrm{A} 01$ & 716 & 1 \\
\hline AT5G11250.1 & TIR-NBS-LRR & Chr5 & 3,982 & 4 & Bra008977 & NBS & A10 & 518 & 1 \\
\hline AT5G45490.1 & NBS & Chr5 & 1,394 & 1 & Bra021980 & NBS & $\mathrm{A} 02$ & 1,130 & 1 \\
\hline AT5G56220.1 & NBS & Chr5 & 3,102 & 1 & Bra002834 & NBS & $\mathrm{A} 10$ & 2,918 & 1 \\
\hline AT1G12210.1 & NBS-LRR & Chr1 & 2,657 & 1 & Bra019755 & NBS-LRR & A06 & 2,682 & 2 \\
\hline \multirow[t]{2}{*}{ AT1G12220.1 } & NBS-LRR & Chr1 & 2,882 & 1 & Bra019754 & NBS-LRR & A06 & 2,672 & 1 \\
\hline & & & & & Bra016311 & NBS-LRR & A08 & 4,678 & 5 \\
\hline AT3G14460.1 & NBS-LRR & Chr3 & 4,274 & 1 & Bra027333 & NBS-LRR & A05 & 4,229 & 1 \\
\hline AT3G14470.1 & NBS-LRR & Chr3 & 3,307 & 1 & Bra027332 & NBS-LRR & A05 & 3,128 & 1 \\
\hline AT4G12020.2 & NBS-LRR & Chr4 & 7,992 & 16 & Bra000758 & NBS-LRR & $\mathrm{A} 03$ & 4,423 & 5 \\
\hline AT4G19050.1 & NBS-LRR & Chr4 & 3,684 & 2 & Bra013372 & NBS-LRR & $\mathrm{A} 01$ & 3,541 & 2 \\
\hline AT4G27190.1 & CC-NBS-LRR & Chr4 & 2,957 & 1 & Bra026368 & NBS-LRR & $\mathrm{A} 01$ & 2,933 & 1 \\
\hline AT4G33300.1 & NBS-LRR & Chr4 & 5,475 & 5 & Bra034556 & NBS-LRR & A08 & 3,140 & 5 \\
\hline \multirow[t]{2}{*}{ AT5G04720.1 } & NBS & Chr5 & 3,172 & 5 & Bra009434 & NBS-LRR & $\mathrm{A} 10$ & 3,120 & 5 \\
\hline & & & & & Bra022036 & NBS-LRR & $\mathrm{A} 02$ & 6,957 & 6 \\
\hline AT5G66900.1 & CC-NBS-LRR & Chr5 & 3,024 & 5 & Bra012116 & NBS-LRR & A07 & 4,738 & 7 \\
\hline AT1G61310.1 & CC-NBS-LRR & Chr1 & 2,880 & 1 & Bra027097 & NBS-NBS-LRR & A09 & 2,736 & 3 \\
\hline AT1G17615.1 & TIR-NBS & Chr1 & 1,226 & 2 & Bra025962 & TIR-NBS & A06 & 1,634 & 2 \\
\hline AT1G72840.1 & TIR-NBS-LRR & Chr1 & 4,529 & 4 & Bra008053 & TIR-NBS & $\mathrm{A} 02$ & 8,376 & 5 \\
\hline AT1G72860.1 & TIR-NBS & Chr1 & 4,550 & 3 & Bra008056 & TIR-NBS & A02 & 1,832 & 2 \\
\hline \multirow[t]{3}{*}{ AT1G72890.1 } & TIR-NBS & Chr1 & 1,770 & 2 & Bra016029 & TIR-NBS & A07 & 1,428 & 2 \\
\hline & & & & & Bra008060 & TIR-NBS & $\mathrm{A} 02$ & 1,685 & 2 \\
\hline & & & & & Bra003864 & TIR-NBS & A07 & 1,661 & 2 \\
\hline AT1G72950.1 & TIR-NBS & Chr1 & 1,395 & 2 & Bra016028 & TIR-NBS & A07 & 1,366 & 2 \\
\hline AT5G45240.1 & TIR-NBS-LRR & Chr5 & 5,383 & 10 & Bra021957 & TIR-NBS & $\mathrm{A} 02$ & 7,454 & 2 \\
\hline AT1G27170.1 & TIR-NBS-LRR & Chr1 & 4,858 & 5 & Bra024651 & TIR-NBS-LRR & A09 & 3,671 & 4 \\
\hline AT1G27180.1 & TIR-TIR-NBS & Chr1 & 6,247 & 6 & Bra016314 & TIR-NBS-LRR & A08 & 4,645 & 5 \\
\hline \multirow[t]{2}{*}{ AT1G63730.1 } & TIR-NBS-LRR & Chr1 & 3,362 & 4 & Bra027791 & TIR-NBS-LRR & A09 & 13,529 & 6 \\
\hline & & & & & Bra003867 & TIR-NBS-LRR & A07 & 6,912 & 10 \\
\hline AT3G51570.1 & TIR-NBS-LRR & Chr3 & 4,098 & 5 & Bra036790 & TIR-NBS-LRR & A09 & 4,182 & 6 \\
\hline
\end{tabular}


Table 3 Orthologous gene pairs of NBS-encoding genes between A. thaliana and B. rapa genomes (Continued)

\begin{tabular}{|c|c|c|c|c|c|c|c|c|c|}
\hline \multirow[t]{2}{*}{ AT4G12010.1 } & TIR-NBS-LRR & Chr4 & 4,182 & 5 & Bra029431 & TIR-NBS-LRR & A09 & 4,646 & 5 \\
\hline & & & & & Bra000759 & TIR-NBS-LRR & A03 & 3,934 & 5 \\
\hline AT4G16890.1 & TIR-NBS-LRR & Chr4 & 4,949 & 7 & Bra012688 & TIR-NBS-LRR & A03 & 5,918 & 9 \\
\hline \multirow[t]{2}{*}{ AT4G19500.1 } & TIR-NBS-TIR-NBS-LRR & Chr4 & 4,736 & 5 & Bra013400 & TIR-NBS-LRR & A01 & 5,825 & 8 \\
\hline & & & & & Bra012540 & TIR-NBS-LRR & A03 & 4,567 & 8 \\
\hline AT4G36150.1 & TIR-NBS-LRR & Chr4 & 3,992 & 5 & Bra011666 & TIR-NBS-LRR & A01 & 4,726 & 6 \\
\hline AT5G17680.1 & TIR-NBS-LRR & Chr5 & 4,154 & 4 & Bra013959 & TIR-NBS-LRR & A08 & 4,066 & 4 \\
\hline \multirow[t]{2}{*}{ AT5G17970.1 } & TIR-NBS-LRR & Chr5 & 2,620 & 4 & Bra002117 & TIR-NBS-LRR & $\mathrm{A} 10$ & 3,627 & 4 \\
\hline & & & & & Bra023647 & TIR-NBS-LRR & A02 & 2,888 & 4 \\
\hline \multirow[t]{2}{*}{ AT5G18350.1 } & TIR-NBS-LRR & Chr5 & 4,500 & 6 & Bra002154 & TIR-NBS-LRR & $\mathrm{A} 10$ & 4,737 & 5 \\
\hline & & & & & Bra006452 & TIR-NBS-LRR & A03 & 8,631 & 9 \\
\hline AT5G41550.1 & TIR-NBS-LRR & Chr5 & 3,553 & 4 & Bra028500 & TIR-NBS-LRR & A07 & 4,012 & 4 \\
\hline AT5G45230.1 & TIR-NBS-LRR & Chr5 & 6,156 & 6 & Bra021956 & TIR-NBS-LRR & A02 & 4,277 & 5 \\
\hline AT5G45250.1 & TIR-NBS-LRR & Chr5 & 4,108 & 5 & Bra027599 & TIR-NBS-LRR & A09 & 3,931 & 5 \\
\hline AT5G46450.1 & TIR-NBS-LRR & Chr5 & 3,928 & 5 & Bra017542 & TIR-NBS-LRR & A09 & 3,362 & 5 \\
\hline AT5G46470.1 & TIR-NBS-LRR & Chr5 & 7,040 & 6 & Bra017544 & TIR-NBS-LRR & A09 & 5,508 & 7 \\
\hline AT1G17600.1 & TIR-NBS-LRR & Chr1 & 3,322 & 4 & Bra030998 & TIR-NBS-LRR-NBS & A09 & 5,997 & 8 \\
\hline AT4G36140.1 & TIR-NBS-TIR-NBS-LRR & Chr4 & 5,523 & 7 & Bra011665 & TIR-NBS-LRR-TIR & A01 & 4,843 & 6 \\
\hline AT5G18370.1 & TIR-NBS-LRR & Chr5 & 3,890 & 4 & Bra002153 & TIR-NBS-NBS-LRR & $\mathrm{A} 10$ & 7,583 & 7 \\
\hline
\end{tabular}

Note: NY, not yet assigned to a chromosome.

\section{Syntenic analysis of orthologous gene pairs for NBS-} encoding genes among $B$. oleracea, $B$. rapa and $A$. thaliana Whether retention of Brassica triplets is random or determined by their genomic position or function remains unknown. We investigated the syntenic relationship of sample region in $A$. thaliana containing four genes compared to syntenic counterpart regions in B. oleracea and $B$. rapa genomes to detect deletion or loss on triplicated regions among 3 species. The genes from AT4G19500 AT4G19530 were found in tandem arrays located on the sample region of chromosome 4 in A. thaliana genome. Only two genes in this tandem array (AT4G19500 and AT4G19510) preserved tripled copies and other two genes (AT4G19520 and AT4G19530) have retained one copy in $B$. oleracea genome respectively. In B. rapa genome, we found that only AT4G19500 gene preserved two copies and other members of this tandem arrays were missed or deleted (Figure 3A). From analysis of orthologous gene pairs, it is clear that this region is three copied region retained in B. oleracea genome and two copied regions in $B$. rapa genome. As to every member of tandem array in
A. thaliana has a corresponding copy on triplicated regions of $B$. oleracea and also has a clear syntenic relationship between two species, we can speculate that this tandem array was generated before the split of $A$. thaliana and Brassica ancestor.

From phylogenetic analysis, it is clear that AT4G19500.1 have three homologous genes (Bol029862, Bol003710 and Bol024375) in B. oleracea and two homologous genes (Bra013400 and Bra012540) in B. rapa, which were clustered in one phylogenetic sub tree corresponding to syntenic relationship. The second member of tandem array, AT4G19510.1 have three homologous genes (Bol024376, Bol003711 and Bol029861) only in B. oleracea and did not retain any copy in $B$. rapa genome, indicating syntenic relationship between two species. Each of tandem array member, AT4G19520.1 and AT4G19530.1 have one homologous genes (Bol024371 and Bol024372) only in $B$. oleracea respectively, which appeared in one phylogenetic sub tree (Figure 3B).

The genes AT4G19500 and AT4G19510 in tandem array might have important role in process of $A$. thaliana

Table 4 Statistics of tandem arrays for NBS-encoding genes in A. thaliana, B. rapa and B. oleracea

\begin{tabular}{|c|c|c|c|c|c|c|c|c|}
\hline Categories & $\begin{array}{c}\text { Total NBS } \\
\text { genes }\end{array}$ & $\begin{array}{c}\text { Tandem } \\
\text { genes }\end{array}$ & Percentage (\%) & $\begin{array}{c}\text { Tandem } \\
\text { arrays }\end{array}$ & $\begin{array}{c}\text { Common } \\
\text { tandem genes }\end{array}$ & $\begin{array}{c}\text { Common } \\
\text { tandem arrays }\end{array}$ & $\begin{array}{l}\text { Located on } \\
\text { chromosomes }\end{array}$ & Unanchored \\
\hline A. thaliana & 167 & 93 & 55.7 & 37 & 20 & 10 & 93 & / \\
\hline B. rapa & 206 & 97 & 47.1 & 40 & 14 & 7 & 93 & 4 \\
\hline B. oleracea & 157 & 68 & 43.3 & 26 & 18 & 9 & 57 & 11 \\
\hline
\end{tabular}




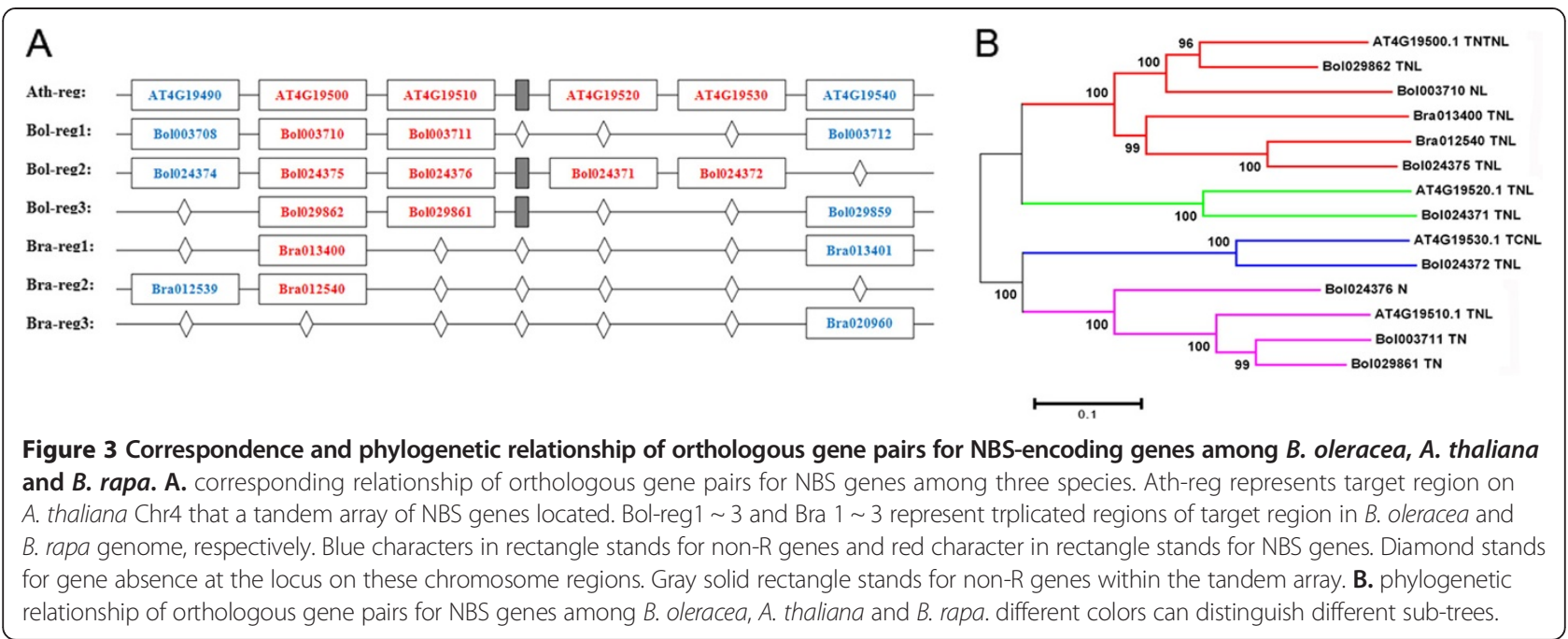

diseases resistance, so they retained three copies after triplication in B. oleracea genome. AT4G19520 and AT4G19530 might have subjected to less evolutionary pressure leading to other two other two duplicated copies lost in B. oleracea genome. We hypothesize that these homologous genes of $B$. oleracea might be resistant to species-specific pathogens or diseases in B. oleracea genome. After WGT of Brassica ancestor, genomic components were triplicated and redundance data was generated. From evolutionary pressure or environment selection, critical components were retained and others were deleted or lost.

Expression analysis of orthologous and paralogous gene pairs for NBS-encoding genes among $B$. oleracea, $B$. rapa and $A$. thaliana

Differential expression level of orthologous and paralogous gene pairs for NBS-encoding genes can reflect expression pattern divergence of orthologous and paralogous genes after WGT. Through syntenic analysis among 3 species, we focused on transcript expression level of 5 CNL and 16 TNL NBS-encoding genes in different tissues in A. thaliana which have their corresponding orthologous and paralogous genes in $B$. rapa and $B$. oleracea genomes to investigate expression pattern divergence among 3 species.

In CNL group in case of orthologs, the expression of two orthologous genes (corresponding to $A$. thaliana gene AT1G50180.1), one in B. oleracea (Bol011780) and one in $B$. rapa (Bra014241), was found to be different across the different tissues. Bol011780 showed reduced expression in stalk, silique and moderately expressed in callus, on the other hand we only observed the reduced expression of Bra014241 in stalk, leaf and flower. Orthologous gene Bol039594 in B. oleracea (corresponding to A. thaliana gene AT4G26090.1) expressed only in root, silique and callus while the expression of its corresponding analogue Bra013947 in B. rapa was confined to stalk and callus. Further, Bol042325 (corresponding to A. thaliana gene AT4G27190.1) expressed in leaf, flower and callus but expression of its orthologous gene in B. rapa (Bra019063) was significantly decreased in all tissues. Another orthologous gene in B. oleracea, Bol005097 (corresponding to A. thaliana gene AT3G14460.1) was abundantly expressed in leaf, callus and moderately expressed in root, stalk and silique and its orthologous gene, Bra027333 in B. rapa displayed high expression in stalk, leaf, flower, callus and reduced expression in root and silique. Bol005098 in $B$. oleracea (corresponding to $A$. thaliana gene AT3G14470.1) abundantly expressed in root, stalk, leaf, callus and exhibited reduced expression in flower and silique, whereas its orthologous gene Bra027332 in $B$. rapa, was ubiquitously expressed in all tissues (Figure 4A). In CNL group, the above mentioned two genes in $A$. thaliana (AT3G14460.1 and AT3G14470.1) are also located in a tandem array and two paralogs in each of B. oleracea (Bol005097 and Bol 005098) and B. rapa (Bra027332 and Bra027333) were generated by their tandem duplication. When we compared the expression profile between these two paralogs (Bol005097 and Bol 005098) in B. oleracea, we found that there was a clear difference in expression level of these two paralogs in different tissues except the root and callus where they transcribe almost at the same level. In $B$. rapa, the expression of Bra027332 and Bra027333 paralogs was significantly high in stalk, leaf, flower and callus, but Bra027332 exhibited moderate and Bra027333 showed low expression level in root and siliques (Figure 4A).

In TNL group in case of orthologs, four orthologs (two in each of $B$. oleracea and $B$. rapa) corresponding to A. thaliana gene AT1G72890.1 have been identified. In B. oleracea Bol026304 was observed to express in stalk, 


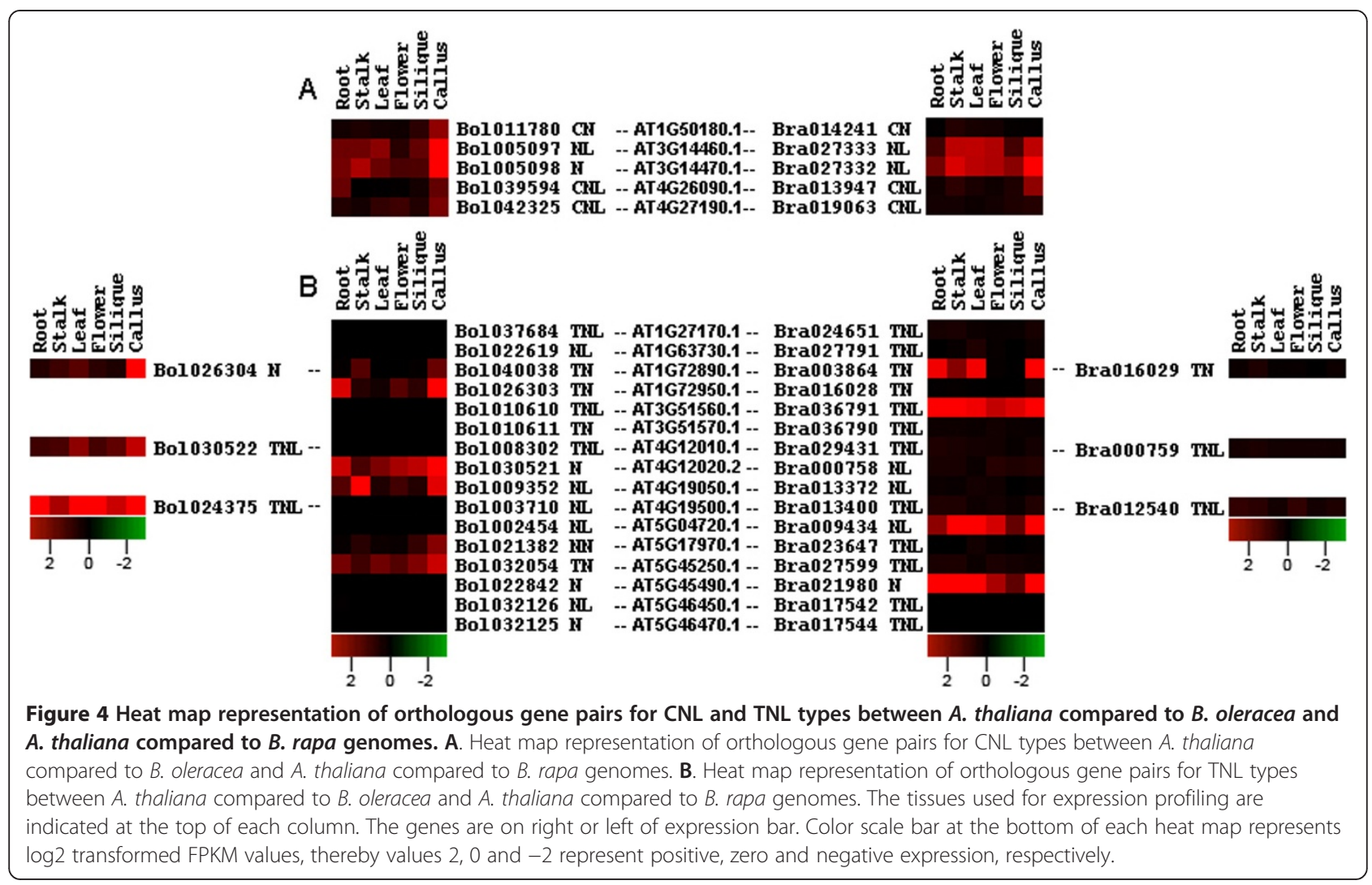

leaf, flower and callus whereas Bol040038 only expressed in stalk and callus. In $B$. rapa one of the retained orthologous copy (Bra003864) expressed in vegetative tissues and other orthologous gene (Bra016029) was down regulated in all tissues. Another A. thaliana gene (AT1G72950.1) retained a single ortholog in each of $B$. oleracea (Bol0 26303) and B. rapa (Bra016028), where Bol026303 was noticed in all tissues but specifically highly expressed in root and callus, while its orthologous gene Bra016028 transcribe at too low level to be detected. In one more case in TNL type, A. thaliana gene AT4G12010.1 retained corresponding two orthologous genes in each of $B$. oleracea (Bol008302 and Bol030522) and B. rapa (Bra029431 and Bra000759). One of the ortholog Bol030522 expressed more or less in all tissues while rest of the orthologs transcribed at too low level. Furthermore, a single copy in each of B. oleracea (Bol030521) and B. rapa (Bra000758) was retained corresponding to $A$. thaliana gene AT4G12020.2. Bol030521 ubiquitously expressed in most of the tissues but the expression level of its ortholog, Bra000758 in B. rapa was very reduced in all tissues (Figure 4B). In TNL group, a tandem array (AT4G12010.1 and AT4G12020.2) in A. thaliana gave rise to three paralogs (two generated by tandem duplication and one by genome triplication) in each of B. oleracea (Bol030521, Bol030522 and Bol008302) and B. rapa (Bra000758, Bra000759 and Bra029431). Through expression profile comparison it is clear that in
B. oleracea, Bol030521 highly expressed in root, leaf, flower, silique and callus, Bol030522 distinctly expressed in leaf, silique and callus while the expression level of Bol008302 was very low across all tissues studied. In $B$. rapa, the expression of two paralogs (Bra000758 and Bra029431) was significantly reduced in all tissues while Bra000759 was detected at very low level. In addition to that the other two genes in A. thaliana, AT1G72890.1 and AT1G72950.1 have also generated three paralogs (again two generated by tandem duplication and one by genome triplication) in B. oleracea and B. rapa. In B. oleracea, Bol026303 was noticeably expressed in root, flower and callus, Bol026304 showed clear expression only in stalk, leaf and callus, whereas the third paralog Bol040038 was only detected in stalk and callus. In B. rapa, Bra003864 showed significantly wide expression in root, stalk, leaf and callus whereas the other two paralogs Bra016028 and Bra016029 exhibited very low expression level in all tissues (Figure 4B).

Through expression divergence analysis in CNL and TNL type by comparing the difference between paralogous and orthologous gene pairs, the results indicate the functional variability of these retained orthologous and paralogous gene copies in B. oleracea and B. rapa. The expression profile diverged more in paralogous than orthologous gene pairs, consequently paralogous genes might contribute more towards functional divergence 
than orthologous genes over evolutionary history in Brassicaceae family.

\section{Comparative evolutionary analyses of orthologous gene} pairs for NBS-encoding genes

$\mathrm{Ka} / \mathrm{Ks}$ value is the ratio between the number of nonsynonymous substitutions per nonsynonymous site $(\mathrm{Ka})$ and the number of synonymous substitution per synonymous site (Ks). The ratios of the rates of nonsynonymous to synonymous substitutions $(\mathrm{Ka} / \mathrm{Ks})$ of orthologous gene pairs were estimated for each branch of the phylogenetic tree using PAML software [40]. The Ka/Ks ratio for orthologous gene pairs was employed to detect the evolutionary selection pattern of NBS-encoding genes among the three species. Through comparative analysis of orthologous gene pairs for NBS-encoding genes among three species, we found $5 \mathrm{CNL}$ and $16 \mathrm{TNL} \mathrm{R}$ genes in A. thaliana which have their corresponding gene copies in A. thaliana compared to B. rapa and $A$. thaliana compared to $B$. oleracea genomes. Out of 16 TNL R genes in A. thaliana, three genes retained two copies. Thus, we obtained 19 orthologous gene pairs in A. thaliana compared to B. rapa and $A$. thaliana compared to $B$. oleracea genomes (Figure 5). For CNL type, the mean $\mathrm{Ka} / \mathrm{Ks}$ ratios of all orthologous genes was 0.497 in B. oleracea genome compared to its recent reconstructed ancestor, which is greater $(52.72 \%)$ than that $(0.235)$ of $B$. rapa genome. The Ka/Ks values of orthologous gene pairs of CNL type $\mathrm{R}$ genes

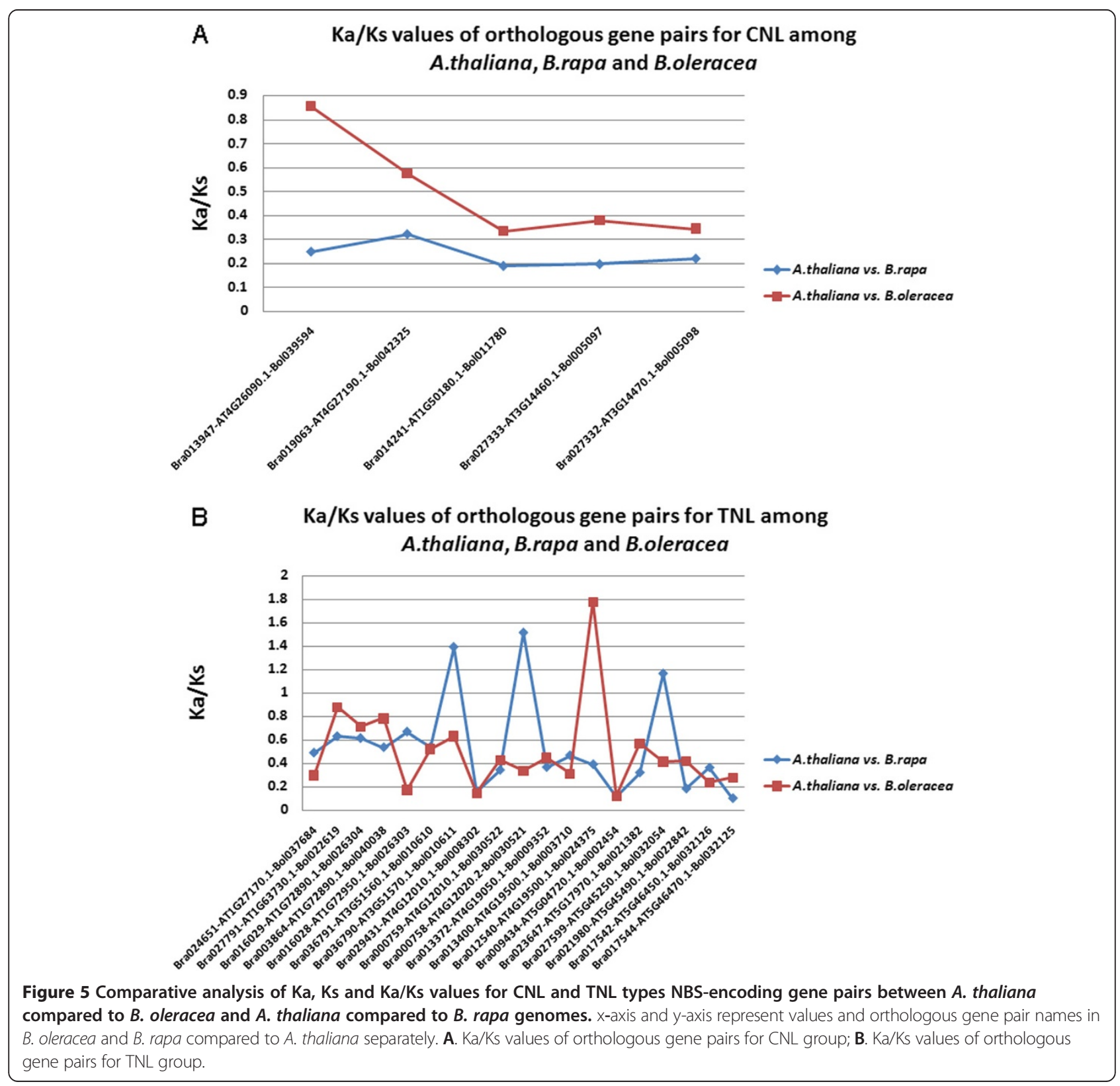


were found to show significant differences in B. oleracea and $B$. rapa species $(\mathrm{P}=0.009<0.05$, Mann-Whitney U-test, Figure 5A) [47]. The mean $\mathrm{Ka} / \mathrm{Ks}$ values of all orthologous gene pairs for TNL group was 0.497 in $B$. oleracea genome, which is slightly lower than $\mathrm{Ka} / \mathrm{Ks}$ value of 0.54 in $B$. rapa genome, but there are no significant differences between the two species $(P=0.759>0.05$, Mann-Whitney U-test, Figure 5B).

Comparing the mean $\mathrm{Ka} / \mathrm{Ks}$ ratios for $\mathrm{CNL}$ group, we observed that orthologous genes of CNL type NBSencoding genes in $B$. rapa species experienced stronger pressure about negative selection than $B$. oleracea species which specify that $B$. oleracea species experienced stronger evolutionary constraints than B. rapa species in CNL type NBS-encoding orthologous genes. But for TNL group, we could not find significant differences between the two species about the orthologous gene pairs, although the mean $\mathrm{Ka} / \mathrm{Ks}$ values of all orthologous gene pairs for TNL group in B. rapa genome was slightly higher than that of $B$. oleracea genome.

\section{Analysis of $\mathbf{R}$ genes with known functions for specific pathogens in $A$. thaliana and Brassica species}

We retrieved the characterized gene-for-gene type NBS proteins in A. thaliana through PRGdb (http://prgdb. crg.eu/wiki) database [4] and their corresponding genes were obtained from TAIR10 [27]. These pathogen resistant genes were compared with conserved orthologous gene pairs in Brassica species and totally four NBS disease resistance genes were obtained in A. thaliana which retained the orthologous gene pairs in Brassica species. In A. thaliana, RPM1 gene enables dual specificity resistance to two avirulence products (AvrRpm1 or AvrB type III effector protein) of Pseudomonas syringae, a causal agent of bacterial blight disease [48]. In the conserved orthologous gene pairs, we found one gene with NBS domain in B. oleracea (Bol008133) analogous to the RPM1. We also recognized one conserved gene, Bra040356 in B. rapa corresponding to RPM1 but its domain type could not identified. Another disease resistance gene, RPS2 in A. thaliana is known to have NBS, LRR and LZ domains and confers resistance to $P$. syringae avirulence gene avrRpt2 [49]. Earlier Wroblewski et al. [50] identified RPS2 homologs with high nucleotide similarity in Brassica species, like B. montana AF180358 (98\% identity), B. oleracea AF180357 (98\% identity) and B. rapa AF180359 (95\% identity). Later Malvas et al. [51] also described the involvement of B. oleracea RPS2 homo$\log$ in disease resistance which is constitutively expressed. In our study, two CNL type NBS genes, one in B. rapa (Bra013947-previous GenBank ID AF180359) and one in B. oleracea (Bol039594- previous GenBank ID AF180357) were found to be conserved parallel to the RPS2 gene with 94.59\% nucleotide similarity. In addition to that both
B. oleracea and B. rapa have retained one copy of (Bra027599) and (Bol032054) genes parallel to RPS4 which was previously recognized to specify resistance to avirulence gene avrRps4 from $P$. syringae pv. Pisi in $A$. thaliana [52]. Two genes only existed in B. rapa (Bra019754 and Bra016781) were preserved with RPS5 disease resistance gene in $A$. thaliana that is responsible for recognizing avirulence gene $a v r P p h B$ from $P$. syringae pv.Phaseolicola [53].

\section{Discussion}

\section{TNL, TN and TX genes in Brassica species}

The present study suggests the abundance of TNL class in Brassicaceae family (A. thaliana, B. oleracea and B. rapa) however, comparisons across plant species demonstrated that in T. cacao, P. trichocarpa, V. vinifera; and M. truncatula the CNL class predominates. In contrast to the NBSencoding genes, the current analysis revealed a higher number of TIR-X (TX-lacking both NBS and LRRs) and TIR-NBS (TN-lacking LRRs) genes in B. oleracea (82 and 29) than A. thaliana (46 and17), B. rapa (42 and 23), Theobroma cacao (17 and 4), Populus trichocarpa (67 and 10) and Vitis vinifera (10 and 14). Furthermore, in three Brassica species (A. thaliana, B. oleracea and B. rapa) the number of both TX and TN genes and only TN genes was found to be higher than T. cacao, V. vinifera and P. trichocarpa respectively. $B$. oleracea despite having a larger genome $(630 \mathrm{Mb})$ contained less NBS genes than $A$. thaliana $(123 \mathrm{Mb})$ and $B$. rapa $(485 \mathrm{Mb})$ which is may be because of prevalence of higher number of TX, TN and $\mathrm{N}$ type genes. TX, TN and TNL genes are prevalent in both conifers and dicots which point out their presence approximately 300 MYA before the divergence of these taxa [54]. The function of TX and TN gene families in plants is unknown yet but their presence in various plant species make them important. Based on the homology of TX and TN genes to the NBS-LRR disease resistance proteins, these shorter proteins act as adapter proteins and have been suggested to play the same role as of Mal and MyD88 (TIR-containing adapter proteins) in the immune system of mammals. In addition, these are also considered as functional proteins and may interact with TNL class for their function in disease resistance [9].

\section{Influences of tandem duplication and whole genome triplication in Brassica species}

Plants have experienced more genome duplication events than any other eukaryotes on the earth. After the duplication, genes can follow one of the three functional outcomes, gene loss, neo-functionalization and sub-functionalization [55]. In duplication events, either it is whole genome, segmental or tandem, some genes have more probability to be retained as duplicates. This may be attributed to the certain function of the duplicated genes in 
particular organism [56,57]. Approximately 43.3\% NBSencoding genes in B. oleracea, $55.7 \%$ in A. thaliana and $47.1 \%$ in B. rapa were formed by tandem duplication and distributed in tandem arrays. Tandem duplicated analysis reveal that the rate of tandem duplicated genes was found to be higher in A. thaliana than Brassica species. After tracing the fate of tandem arrays before or after complete genome triplication, 50 NBS genes were tandem duplicated genes after divergence of $B$. oleracea and B. rapa species, representing $31.8 \%$ of total NBS-encoding genes in $B$. oleracea genome. There are 83 NBS genes generated after divergence of $B$. oleracea and $B$. rapa species, which represents $40.3 \%$ of all NBS genes in $B$. rapa genome. Through analysis of whole genome triplication, $42 \mathrm{~B}$. oleracea and $62 \mathrm{~B}$. rapa NBS genes were retained on triplicated regions in genomes, representing $26.75 \%$ and $30.1 \%$ of total NBS genes in B. oleracea and B. rapa genomes, respectively.

Polyploidy event provide rich genomic resources to study retention and loss of multi-copy genes. Through comparative analysis of NBS-encoding genes among B. oleracea, B. rapa and $A$. thaliana, we speculated that after WGT of Brassica ancestor approximately 16 MYA, orthologous gene pairs for NBS-encoding genes on triplicated regions in genome of Brassica ancestor were lost or deleted quickly. The quick loss of paralogs from whole genome duplication might be due to the gene dosage imbalance issue $[58,59]$. But after divergence of B. rapa and B. oleracea approximately 3.7 MYA, NBSencoding genes in Brassica species experienced speciesspecific gene amplification by mechanism of tandem duplication to adopt environment selection. The increase of gene dosage by tandem paralogs might have some advantage to the plant pathogen defense.

\section{Disease resistance in Brassica species}

To environmentally friendly control plant diseases, one of the efficient methods is the employment of genetically disease resistant plants. In the last few years, the studies regarding disease resistance inheritance and molecular cloning of R genes in Brassica species made some progress. We found eight conserved genes in Brassica species corresponding to the four A. thaliana R genes (RPM1, RPS2, RPS4 and RPS5) species in the conserved orthologous region. In present study, we observed one ( $\mathrm{N}$ type) gene in $B$. oleracea and two (CNL type) genes in B. oleracea and B. rapa corresponding to RPM1 and RPS2 respectively. Therefore, we propose that these conserved genes in the Brassica species may offer gene-to-gene resistance to specific avirulence products from Pseudomonas syringae pathogen. A. thaliana gene RPS4 (AT5G45250) retained one corresponding orthologous gene in $B$. oleracea (Bol032054) and B. rapa (Bra027599) genomes respectively, so we assume that these two genes in Brassica species might be race-specific resistant to Pisi and Phaseolicola subspecies of Pseudomonas syringae pathogen. RPS5 retained two copies only in $B$. rapa and these gene copies are species-specific disease resistance genes in $B$. rapa species.

For evolutionary relationship of orthologous gene pairs for race-specific NBS-encoding genes among three species, we compared $\mathrm{Ka} / \mathrm{Ks}$ values of orthologous gene pairs between $A$. thaliana - B. rapa and A. thaliana $B$. oleracea lineages. A. thaliana - B. oleracea lineage exhibited higher mean $\mathrm{Ka} / \mathrm{Ks}$ ratios in their orthologous gene pairs than those of $A$. thaliana - B. rapa lineage in CNL type NBS-encoding $\mathrm{R}$ genes. We conclude that these NBS-encoding genes in $B$. rapa species have undergone stronger negative selection than those in $B$. oleracea species. So, the corresponding NBS-encoding genes in $B$. oleracea species would have experienced stronger evolutionary constraints to adopt changes in the environment. But for TNL type, there are no significant differences between the two species about the orthologous gene pairs. We speculated that these NBSencoding genes in B. rapa and $B$.oleracea species may have undergone different selection pressure to offer resistance to same pathogen or some pathogens may be species-specific pathogens for Brassica species.

\section{Conclusions}

We have identified 157, 206 and 167 NBS-encoding genes in $A$. thaliana, B. rapa and B. oleracea genomes respectively and total number of NBS-encoding genes in these three species is very close in spite of genome size and WGD/WGT events. Genomic organization and composite phylogenetic analysis facilitate the identification and classification of NBS-encoding genes among A. thaliana, $B$. rapa and $B$. oleracea. Expression profiling showing the differential expression pattern of orthologous NBSencoding genes provides a blueprint for further characterization of these genes in $B$. oleracea and $B$. rapa. The expression profile of different NBS-coding members can be separated into different groups, indicative of functional divergence. Although, orthologous NBS-encoding genes in B. oleracea and B. rapa are highly divergent but expression pattern divergence among paralogs within a species exceeds the level of divergence among orthologs in each type of NBS-encoding genes. Paralogs might contribute more to functional divergence than orthologs over the evolution of Brassicaceae. Through comparative analysis of tandem duplication and whole genome triplication in NBS-encoding genes among three species, there are fewer paralogous NBS-encoding genes retention after whole genome triplication than those from tandem duplication. So, tandem duplication might play more important influence than whole genome duplication in generation of NBS-encoding genes. We speculated that the quick loss of 
paralogs from whole genome duplication might be due to the gene dosage imbalance issue. The increase of gene dosage by tandem paralogs might have some advantage to the plant pathogen defense. Our evolutionary studies illustrate that CNL type orthologous genes in $B$. rapa species compared to $A$. thaliana have undergone stronger negative selection than those in B. oleracea species compared to $A$. thaliana and opposite to that orthologous genes in $B$. oleracea species experienced stronger evolutionary constraints than those in $B$. rapa species for CNL type $\mathrm{R}$ genes. For TNL type NBS-encoding genes, we did not observed significant difference between the two species about the orthologous gene pairs using Mann-Whitney U-test. It is indicated that these orthologous NBSencoding genes in $B$. rapa and $B$.oleracea species maybe undergone different selection pressure to resist the same pathogen or some pathogens may act as species-specific pathogens for different Brassica species. Through comparative analysis of NBS-encoding genes among $A$. thaliana, B. rapa and B. oleracea, we hope to explore the evolutionary fate of NBS-encoding genes in Brassica lineage after split from Arabidopsis thaliana and advance the understanding of disease resistance between $B$. oleracea and $B$. rapa species, which will provide a valuable model for studying functional and evolutionary aspects within the Brassica genus and the crucifer lineage.

\section{Additional files}

Additional file 1: Table S1. Information of NBS-encoding genes in B. oleracea, B. rapa and A. thaliana. This table contain the type, distribution of NBS domains, protein full length, subfamily, lists of predicted domains, coding sequences and peptide sequences of NBS-encoding genes in B. oleracea, B. rapa and A. thaliana genomes.

Additional file 2: Figure S1. Phylogenetic relationship of NBSencoding genes among $B$. oleracea, $A$. thaliana and $B$. rapa. The Maximum Likelihood tree was constructed by MEGA 5.0 software with 1000 replications. CNL type of NBS-encoding genes was divided into three sub-groups and TNL type was divided into three sub-groups. Each species was shown by different colors.

Additional file 3: Figure S2. Heat map representation of NBS-encoding genes in B. oleracea and B. rapa genomes. I. Heat map representation of NBS-encoding genes in B. oleracea genomes. II. Heat map representation of NBS-encoding genes in $B$. rapa genomes. The tissues used for expression profiling are indicated at the top of each column. The genes are on right expression bar. Color scale bar at the bottom of each heat map represents $\log 2$ transformed FPKM values, thereby values more than 2, 0 and less than -2 represent positive, zero and negative expression, respectively.

Additional file 4: Table S2. List of Tandem arrays of NBS-encoding genes among B. oleracea, B. rapa and A. thaliana. This table contain name, location, gene numbers, gene lists of tandem arrays in $B$. oleracea, B. rapa and $A$. thaliana genomes.

Additional file 5: Table S3. Co-retained tandem duplicated genes of NBS-encoding genes in A. thaliana compared to B. oleracea and A. thaliana compared to $B$. rapa genomes. This table contain co-retained tandem duplicated genes of NBS-encoding genes in $A$. thaliana compared to B. oleracea and $A$. thaliana compared to B. rapa genomes.

\section{Competing interests}

The authors declare that they have no competing interests.

\section{Authors' contributions}

$J Y$ and ST analyzed the data and prepared the manuscript. SL revised the manuscript. $F Z, C T, J H, X C, C D, Y Z, R Q$ and $W H$ participated data analysis and the manuscript preparation. All authors read and approved the final manuscript.

\section{Acknowledgements}

This work was supported by grants from National Basic Research Program of China (973 program, 2011CB109305), National Natural Science Foundation of China (no. 31301039), National High Technology Research and Development Program of China (863 Program, 2013AA102602),

Commonweal Specialized Research Fund of China Agriculture (201103016), Core Research Budget of the Non-profit Governmental Research Institution (no. 1610172011011) and Hubei Agricultural Science and Technology Innovation Center of China.

\section{Author details}

'Key Laboratory of Biology and Genetic Improvement of Oil crops, the Ministry of Agriculture, Oil Crops Research Institute of the Chinese Academy of Agricultural Sciences, Wuhan 430062, China. ${ }^{2}$ Engineering Research Center of Protection and Utilization for Biological Resources in Minority Regions, South-Central University for Nationalities, Wuhan 473061, China.

Received: 30 June 2013 Accepted: 30 December 2013

Published: 3 January 2014

\section{References}

1. Dangl JL, Jones JD: Plant pathogens and integrated defence responses to infection. Nature 2001, 411(6839):826-833.

2. Anderson JP, Gleason CA, Foley RC, Thrall PH, Burdon JB, Singh KB: Plants versus pathogens: an evolutionary arms race. Funct Plant Biol 2010, 37(6):499-512

3. Vergne E, Grand X, Ballini E, Chalvon V, Saindrenan P, Tharreau D, Nottéghem J, Morel J: Preformed expression of defense is a hallmark of partial resistance to rice blast fungal pathogen Magnaporthe oryzae. BMC Plant Biol 2010, 10(1):206.

4. Sanseverino W, Hermoso A, D'Alessandro R, Vlasova A, Andolfo G, Frusciante L, Lowy E, Roma G, Ercolano MR: PRGdb 2.0: towards a community-based database model for the analysis of R-genes in plants. Nucleic Acids Res 2013, 41(Database issue):D1167-1171.

5. Martin GB, Bogdanove AJ, Sessa G: Understanding the functions of plant disease resistance proteins. Annu Rev Plant Biol 2003, 54:23-61.

6. van Ooijen G, van den Burg HA, Cornelissen BJ, Takken FL: Structure and function of resistance proteins in solanaceous plants. Annu Rev Phytopathol 2007, 45:43-72.

7. Wan H, Yuan W, Ye Q, Wang R, Ruan M, Li Z, Zhou G, Yao Z, Zhao J, Liu S, Yang Y: Analysis of TIR- and non-TIR-NBS-LRR disease resistance gene analogous in pepper: characterization, genetic variation, functional divergence and expression patterns. BMC Genomics 2012, 13:502.

8. Inohara N, Chamaillard M, MCDonald C, Nunez G: NOD-LRR proteins: role in host-microbial interactions and inflammatory disease. Annu Rev Biochem 2005, 74:355-383.

9. Meyers BC, Morgante M, Michelmore RW: TIR-X and TIR-NBS proteins: two new families related to disease resistance TIR-NBS-LRR proteins encoded in Arabidopsis and other plant genomes. Plant J 2002, 32(1):77-92.

10. Meyers BC, Kozik A, Griego A, Kuang H, Michelmore RW: Genome-wide analysis of NBS-LRR-encoding genes in Arabidopsis. Plant Cell 2003, 15(4):809-834.

11. Monosi B, Wisser RJ, Pennill L, Hulbert SH: Full-genome analysis of resistance gene homologues in rice. Theor Appl Genet 2004, 109(7):1434-1447

12. Kohler A, Rinaldi C, Duplessis S, Baucher M, Geelen D, Duchaussoy F, Meyers BC, Boerjan W, Martin F: Genome-wide identification of NBS resistance genes in Populus trichocarpa. Plant Mol Biol 2008, 66(6):619-636.

13. Ameline-Torregrosa C, Wang BB, O'Bleness MS, Deshpande S, Zhu H, Roe B, Young ND, Cannon SB: Identification and characterization of nucleotide-binding site-leucine-rich repeat genes in the model plant Medicago truncatula. Plant Physiol 2008, 146(1):5-21.

14. Porter BW, Paidi M, Ming R, Alam M, Nishijima WT, Zhu YJ: Genome-wide analysis of Carica papaya reveals a small NBS resistance gene family. Mol Genet Genomics 2009, 281(6):609-626. 
15. Yang S, Zhang X, Yue JX, Tian D, Chen JQ: Recent duplications dominate NBS-encoding gene expansion in two woody species. Mol Genet Genomics 2008, 280(3):187-198.

16. Li X, Cheng Y, Ma W, Zhao Y, Jiang H, Zhang M: Identification and characterization of NBS-encoding disease resistance genes in Lotus japonicus. Plant Systemat Evol 2010, 289(1-2):101-110.

17. Zhou T, Wang Y, Chen JQ, Araki H, Jing Z, Jiang K, Shen J, Tian D: Genomewide identification of NBS genes in japonica rice reveals significant expansion of divergent non-TIR NBS-LRR genes. Mol Genet Genomics 2004, 271(4):402-415.

18. Kim J, Lim CJ, Lee BW, Choi JP, Oh SK, Ahmad R, Kwon SY, Ahn J, Hur CG: A genome-wide comparison of NB-LRR type of resistance gene analogs (RGA) in the plant kingdom. Mol Cells 2012, 33(4):385-392.

19. Meyers BC, Dickerman AW, Michelmore RW, Sivaramakrishnan S, Sobral BW, Young ND: Plant disease resistance genes encode members of an ancient and diverse protein family within the nucleotide-binding superfamily. Plant J 1999, 20(3):317-332.

20. Hulbert SH, Webb CA, Smith SM, Sun Q: Resistance gene complexes: evolution and utilization. Annu Rev Phytopathol 2001, 39:285-312

21. Jupe F, Pritchard L, Etherington GJ, MacKenzie K, Cock PJ, Wright F, Sharma SK, Bolser D, Bryan GJ, Jones JD: Identification and localisation of the NB-LRR gene family within the potato genome. BMC Genomics 2012, 13(1):75.

22. Town CD, Cheung F, Maiti R, Crabtree J, Haas BJ, Wortman JR, Hine EE, Althoff R, Arbogast TS, Tallon LJ, Vigourouxb M, Trick M, Bancroft I: Comparative genomics of Brassica oleracea and Arabidopsis thaliana reveal gene loss, fragmentation, and dispersal after polyploidy. Plant Cell 2006, 18(6):1348-1359.

23. Yang TJ, Kim JS, Kwon SJ, Lim KB, Choi BS, Kim JA, Jin M, Park JY, Lim MH, Kim HI, Lim YP, Kang JJ, Hong JH, Kim CB, Bhak J, Bancroft I, Park BS: Sequencelevel analysis of the diploidization process in the triplicated FLOWERING LOCUS C region of Brassica rapa. Plant Cell 2006, 18(6):1339-1347.

24. Blanc $\mathrm{G}$, Hokamp K, Wolfe KH: A recent polyploidy superimposed on older large-scale duplications in the Arabidopsis genome. Genome Res 2003, 13(2):137-144

25. Lysak MA, Koch MA, Pecinka A, Schubert I: Chromosome triplication found across the tribe Brassiceae. Genome Res 2005, 15(4):516-525.

26. UN: Genome analysis in Brassica with special reference to the experimental formation of B. napus and peculiar mode of fertilization. Japan J Bot 1935, 7:389-452.

27. Huala E, Dickerman AW, Garcia-Hernandez M, Weems D, Reiser L, LaFond F, Hanley D, Kiphart D, Zhuang M, Huang W, Mueller LA, Bhattacharyya D, Bhaya D, Sobral BW, Beavis W, Meinke DW, Town CD, Somerville C, Rhee SY: The Arabidopsis Information Resource (TAIR): a comprehensive database and webbased information retrieval, analysis, and visualization system for a model plant. Nucleic Acids Res 2001, 29(1):102-105.

28. Cheng F, Liu S, Wu J, Fang L, Sun S, Liu B, Li P, Hua W, Wang X: BRAD, the genetics and genomics database for Brassica plants. BMC Plant Biol 2011 11:136.

29. Yu J, Zhao M, Wang X, Tong C, Huang S, Tehrim S, Liu Y, Hua W, Liu S: Bolbase: a comprehensive genomics database for Brassica oleracea. BMC Genomics 2013, 14(1):664.

30. Punta M, Coggill PC, Eberhardt RY, Mistry J, Tate J, Boursnell C, Pang N Forslund K, Ceric G, Clements J, Heger A, Holm L, Sonnhammer ELL, Eddy SR, Bateman A, Finn RD: The Pfam protein families database. Nucleic Acids Res 2012, 40(Database issue):D290-301.

31. Finn RD, Clements J, Eddy SR: HMMER web server: interactive sequence similarity searching. Nucleic Acids Res 2011, 39(Web Server issue):W29-37.

32. Thompson JD, Higgins DG, Gibson TJ, Clustal W: improving the sensitivity of progressive multiple sequence alignment through sequence weighting, position-specific gap penalties and weight matrix choice. Nucleic Acids Res 1994, 22(22):4673-4680.

33. McDonnell AV, Jiang T, Keating AE, Berger B: Paircoil2: improved prediction of coiled coils from sequence. Bioinformatics 2006, 22(3):356-358.

34. Delorenzi M, Speed T: An HMM model for coiled-coil domains and a comparison with PSSM-based predictions. Bioinformatics 2002, 18(4):617-625.

35. Ferraiolo J, Jun F, Jackson D: Scalable Vector Graphics (SVG) 1.1 Specification. 2003. http://www.w3.org/TR/SVG11/.

36. Altschul SF, Madden TL, Schaffer AA, Zhang J, Zhang Z, Miller W, Lipman DJ: Gapped BLAST and PSI-BLAST: a new generation of protein database search programs. Nucleic Acids Res 1997, 25(17):3389-3402.
37. Clamp M, Cuff J, Searle SM, Barton GJ: The Jalview Java alignment editor. Bioinformatics 2004, 20(3):426-427.

38. Tamura K, Peterson D, Peterson N, Stecher G, Nei M, Kumar S: MEGA5: molecular evolutionary genetics analysis using maximum likelihood, evolutionary distance, and maximum parsimony methods. Mol Biol Evol 2011, 28(10):2731-2739.

39. Tang H, Wang $X$, Bowers JE, Ming R, Alam M, Paterson AH: Unraveling ancient hexaploidy through multiply-aligned angiosperm gene maps. Genome Res 2008, 18(12):1944-1954.

40. Yang Z: PAML 4: phylogenetic analysis by maximum likelihood. Mol Biol Evol 2007, 24(8):1586-1591.

41. Eisen MB, Spellman PT, Brown PO, Botstein D: Cluster analysis and display of genome-wide expression patterns. Proc Natl Acad Sci USA 1998, 95(25):14863-14868.

42. Mun JH, Yu HJ, Park S, Park BS: Genome-wide identification of NBS-encoding resistance genes in Brassica rapa. Mol Genet Genomics 2009, 282(6):617-631.

43. Friedman AR, Baker BJ: The evolution of resistance genes in multi-protein plant resistance systems. Curr Opin Genet Dev 2007, 17(6):493-499.

44. Richly E, Kurth J, Leister D: Mode of amplification and reorganization of resistance genes during recent Arabidopsis thaliana evolution. $\mathrm{Mol} \mathrm{BiO}$ Evol 2002, 19(1):76-84.

45. Bowers JE, Chapman BA, Rong J, Paterson AH: Unravelling angiosperm genome evolution by phylogenetic analysis of chromosomal duplication events. Nature 2003, 422(6930):433-438.

46. Krzywinski M, Schein J, Birol I, Connors J, Gascoyne R, Horsman D, Jones SJ, Marra MA: Circos: an information aesthetic for comparative genomics. Genome Res 2009, 19(9):1639-1645.

47. Tallarida RJ, Murray RB: Mann-Whitney test, Manual of pharmacologic calculations. New York: Springer; 1986:149-153.

48. Tornero P, Chao RA, Luthin WN, Goff SA, Dangl JL: Large-scale structurefunction analysis of the Arabidopsis RPM1 disease resistance protein. Plant Cell 2002, 14(2):435-450.

49. Bent AF, Kunkel BN, Dahlbeck D, Brown KL, Schmidt R, Giraudat J, Leung J, Staskawicz BJ: RPS2 of Arabidopsis thaliana: a leucine-rich repeat class of plant disease resistance genes. Science 1994, 265(5180):1856-1860.

50. Wroblewski T, Coulibaly S, Sadowski J, Quiros CF: Variation and phylogenetic utility of the Arabidopsis thaliana Rps2 homolog in various species of the tribe Brassiceae. Mol Phylogenet Evol 2000, 16(3):440-448.

51. Malvas CCMM, Truffi D, Camargo LE: A homolog of the RPS2 disease resistance gene is constitutively expressed in Brassica oleracea. Genet Mol Biol 2003, 26(4):511-516.

52. Gassmann W, Hinsch ME, Staskawicz BJ: The Arabidopsis RPS4 bacterialresistance gene is a member of the TIR-NBS-LRR family of diseaseresistance genes. Plant J 1999, 20(3):265-277.

53. Warren RF, Henk A, Mowery P, Holub E, Innes RW: A mutation within the leucine-rich repeat domain of the Arabidopsis disease resistance gene RPS5 partially suppresses multiple bacterial and downy mildew resistance genes. Plant Cell 1998, 10(9):1439-1452.

54. Savard L, Li P, Strauss SH, Chase MW, Michaud M, Bousquet J: Chloroplast and nuclear gene sequences indicate late Pennsylvanian time for the last common ancestor of extant seed plants. Proc Natl Acad Sci USA 1994, 91(11):5163-5167.

55. Force A, Lynch M, Pickett FB, Amores A, Yan YL, Postlethwait J: Preservation of duplicate genes by complementary, degenerative mutations. Genetics 1999, 151(4):1531-1545.

56. Papp B, Pal C, Hurst LD: Dosage sensitivity and the evolution of gene families in yeast. Nature 2003, 424(6945):194-197.

57. Lynch M, Conery JS: The evolutionary fate and consequences of duplicate genes. Science 2000, 290(5494):1151-1155.

58. Freeling M: The evolutionary position of subfunctionalization, downgraded. Genome Dyn 2008, 4:25-40.

59. Freeling M: Bias in plant gene content following different sorts of duplication: tandem, whole-genome, segmental, or by transposition. Annu Rev Plant Biol 2009, 60:433-453.

doi:10.1186/1471-2164-15-3

Cite this article as: Yu et al:: Genome-wide comparative analysis of NBS-encoding genes between Brassica species and Arabidopsis thaliana. BMC Genomics 2014 15:3. 